\title{
TIME-DEPENDENT SCATTERING THEORY OF $N$-BODY QUANTUM SYSTEMS
}

W. HUNZIKER

Institut für Theoretische Physik, ETH-Zürich

E-mail: hunziker@itp.phys.ethz.ch

I. M. SIGAL

Department of Mathematics, University of Toronto

E-mail: sigal@math.toronto.edu

Received 10 April 1998

\begin{abstract}
We give a full and self contained account of the basic results in $N$-body scattering theory which emerged over the last ten years: The existence and completeness of scattering states for potentials decreasing like $r^{-\mu}, \mu>\sqrt{3}-1$. Our approach is a synthesis of earlier work and of new ideas. Global conditions on the potentials are imposed only to define the dynamics. Asymptotic completeness is derived from the fact that the mean square diameter of the system diverges like $t^{2}$ as $t \rightarrow \pm \infty$ for any orbit $\psi_{t}$ which is separated in energy from thresholds and eigenvalues (a generalized version of Mourre's theorem involving only the tails of the potentials at large distances). We introduce new propagation observables which considerably simplify the phase-space analysis. As a topic of general interest we describe a method of commutator expansions.
\end{abstract}

\section{Introduction}

$N$-body quantum systems are described by the Schrödinger equation $i \partial_{t} \psi_{t}=H \psi_{t}$, with a Hamiltonian like

$$
H=\sum_{k=1}^{N} \frac{p_{k}^{2}}{2 m_{k}}+\sum_{i<k}^{1 \cdots N} V_{i k}\left(x_{i}-x_{k}\right) .
$$

After fixing the center of mass $H$ acts on the Hilbert space $\mathcal{H}=L^{2}(X)$, where

$$
X=\left\{x=\left(x_{1}, \ldots, x_{N}\right) \mid x \in R^{3} ; \sum m_{k} x_{k}=0\right\} .
$$

In general $H$ possesses eigenvectors (stationary states), which span the subspace $\mathcal{H}_{B}$ of bound states where the orbits $\psi_{t}$ are recurrent. In the continuous spectral subspace $\mathcal{H}_{C}=\mathcal{H}_{B}^{\perp}$ the long-time behaviour of $\psi_{t}$ depends critically on the decay rate of the potentials, expressed by

$$
V_{i k}\left(x_{i}-x_{k}\right)=O\left(\left|x_{i}-x_{k}\right|^{-\mu}\right) \quad \text { as }\left|x_{i}-x_{k}\right| \rightarrow \infty .
$$

If $\mu$ is not too small it is expected that along any orbit $\psi_{t}$ in $\mathcal{H}_{C}$ the system eventually breaks up into a collection of almost freely moving, bound subsystems (fragments) as $t \rightarrow \pm \infty$. This is the conjecture of asymptotic completeness, which was stated precisely in the early days of scattering theory $[29,22]$. The challenge to

\section{3}

Reviews in Mathematical Physics, Vol. 12, No. 8 (2000) 1033-1084

(c) World Scientific Publishing Company 
prove this conjecture then became a driving force in the mathematical theory of $N$-body systems. New methods of spectral analysis and new tools to control the space-time propagation of quantum states gradually emerged over the last 30 years, often with results not directly related to scattering theory but important in their own right. As a culmination of these efforts Sigal and Soffer [44] in 1987 gave the first general proof of asymptotic completeness for short-range potentials $(\mu>1)$. Further insight and important simplifications came from the subsequent work of Graf [17] and Yafaev [53]. An approach to the qualitatively different long-range problem $(\mu \leq 1)$ was initiated by Sigal and Soffer in their study of the Coulomb case $[46,47]$. With this starting point the long-range problem was solved by Dereziński [9] for $\mu>\sqrt{3}-1$ and by Sigal and Soffer [48] for the Coulomb case $(\mu=1)$.

We briefly summarize the long history of the subject. A first milestone was Faddeev's solution of the 3-body problem using stationary methods (Faddeev equations [16]), later extended to all $N[24,42]$. This approach is used today for computational work on small systems, but its scope, especially for $N>3$, is still limited by spectral assumptions on the subsystems (no eigenvalues embedded in the continuum, no resonances at thresholds). The Faddeev equations and their generalizations are designed to obtain the scattering amplitudes ( $S$-matrix elements in $p$-space). Asymptotic completeness (unitarity of the $S$-matrix) emerges as a by-product at the end, thus offering little intuitive insight into the basic reasons for its validity.

The space-time point of view was primarily developed in quantum field theory under the influence of Haag $[20,21]$ and Ruelle [40]. It regained attention in non-relativistic quantum mechanics after Ruelle's ergodic characterization of bound states vs. continuum states [41] (RAGE Theorem, see e.g. [5] or [26]). Asymptotic completeness for $N$-body systems in the limit of weak forces was obtained by time-dependent perturbation methods $([25,30])$. Positive commutators $([38,32])$ and related (global) propagation estimates first entered in Lavine's proof of asymptotic completeness for $N$-body systems with purely repulsive forces [33, 34]. The commutator in question is familiar from the virial theorem:

$$
i[H, A]=\sum_{k} \frac{p_{k}^{2}}{m_{k}}-\sum_{i<k}\left(x_{i}-x_{k}\right) \cdot \nabla V_{i k}\left(x_{i}-x_{k}\right),
$$

where

$$
A=\sum_{k} \frac{1}{2}\left(x_{k} \cdot p_{k}+p_{k} \cdot x_{k}\right)
$$

For repulsive forces the expression (0.3) is termwise positive. We also note that $A$ is the generator of the unitary group of dilations

$$
e^{-i A \lambda}: \psi(x) \rightarrow e^{-\lambda / 2 \operatorname{dim}(X)} \psi\left(e^{-\lambda} x\right)
$$

acting on $L^{2}(X)$, and that $(0.3)$ is the infinitesimal form of the transformation law

$$
e^{i A \lambda} H e^{-i A \lambda}=H(\lambda):=e^{-2 \lambda} \sum_{k} \frac{p_{k}^{2}}{2 m_{k}}+\sum_{i<k} V_{i k}\left(e^{\lambda}\left(x_{i}-x_{k}\right)\right) .
$$


Moreover, $A$ itself is a commutator, i.e.

$$
A=i\left[H, \frac{1}{2} x^{2}\right], \quad \text { where } \quad x^{2}=\sum_{k} m_{k} x_{k}^{2},
$$

so that

$$
\partial_{t}^{2}\left\langle\frac{1}{2} x^{2}\right\rangle_{t}=\partial_{t}\langle i[H, A]\rangle_{t}>0 .
$$

Here $\langle\Phi\rangle_{t}=\left(\psi_{t}, \Phi \psi_{t}\right)$ denotes the expectation value of an observable $\Phi$ in the state $\psi_{t}$. (0.6) indicates that $\left\langle x^{2}\right\rangle_{t}$ diverges like $t^{2}$ as $t \rightarrow \pm \infty$, which holds in fact for a dense set of initial states $\psi$. A classical example is the case of Coulomb forces: then $i[H, A]=2 H-V \geq H$, where $V$ is the total potential. Therefore, if $\langle H\rangle_{0}>0$, then

$$
\left\langle x^{2}\right\rangle_{t} \geq\langle H\rangle_{0} \cdot t^{2}+O(t) \rightarrow \infty \quad(t \rightarrow \pm \infty)
$$

At the same time and seemingly unrelated to the work of Lavine, Balslev and Combes [3] determined the spectral properties of the operator family $H(\lambda)$ given by (0.5) for complex $\lambda$ in the case of dilation-analytic potentials, thereby revealing the general nature of the essential spectrum of $H$ (thresholds, embedded eigenvalues, absence of singular continuous spectrum) and laying the foundation of a theory of resonances [49]. Further insight came from the geometric (configuration space) methods of spectral analysis and scattering theory developed in the later 1970's, e.g. in $[12,50,7,43])$. The most striking event of that time was Enss' proof that asymptotic completeness for $N=2$ follows directly from Ruelle's Theorem combined with the propagation properties of free wave packets [13]. Although the hope for a quick solution of the general case was premature, Enss' proof (and its later extensions to $N=3$ in $[14,15])$ marks the turning point to phase space analysis in $N$-body scattering theory.

Less noticed at first, and influenced by the work of Lavine and Balslev-Combes, Mourre [35] introduced another key idea: For $N=3$ he proved the conditional positivity of the commutator (0.3) for forces of arbitrary sign, in the sense that

$$
E_{\Delta}(H) i[H, A] E_{\Delta}(H) \geq \theta E_{\Delta}(H)
$$

(Mourre's inequality). Here $E_{\Delta}(H)$ is the spectral projection of $H$ for an energy shell $\Delta=(E-\epsilon, E+\epsilon)$. If $E$ is in the continuous spectrum of $H$, but not an eigenvalue nor a threshold, then (0.7) holds for sufficiently small $\epsilon>0$ with a strictly positive $\theta$. Mourre's inequality again leads to

$$
\left\langle x^{2}\right\rangle_{t} \geq \theta t^{2}+O(t) \rightarrow \infty \quad(t \rightarrow \pm \infty)
$$

for a dense set of initial states in $\mathcal{H}_{\Delta}=\operatorname{Ran}\left(E_{\Delta}(H)\right)$. In fact $(0.7)$ is a special case of a more general inequality given by Mourre, which holds for any $E \in R$, and which exhibits the structure of the essential spectrum of $H$ in much the same way as dilation analyticity (of which it is an infinitesimal version). Mourre's inequality was soon extended to general $N$ by Perry, Sigal and Simon [37], who used it to 
derive the local decay estimate

$$
\int_{-\infty}^{+\infty}\left\langle\left(1+x^{2}\right)^{-\alpha}\right\rangle_{t} \leq C(\Delta, \alpha)\|\psi\|^{2}
$$

for $\alpha>1 / 2$ and all $\psi$ in $\mathcal{H}_{\Delta}$. For an exposition of these results we refer to [5] and [26].

Propagation estimates like (0.8) and (0.9) only say that the mean square diameter $\left\langle x^{2}\right\rangle_{t}$ of the system diverges like $t^{2}$ as $t \rightarrow \pm \infty$. To demonstrate the break-up of the system into fragments (various scenarios of such break-ups are called the scattering channels), it is necessary to show that the probability for it to cross the phase-space boundaries of the channels is relatively small. More precisely, one proves estimates of the form

$$
\int_{-\infty}^{+\infty}\left\|f(x, p) \psi_{t}\right\|^{2} d t \leq C(\Delta, f)\|\psi\|^{2}
$$

for all $\psi$ in $\mathcal{H}_{\Delta}$, where $f$ is $C_{0}^{\infty}$ in $p=\left(p_{1}, \ldots, p_{N}\right)$, smooth and homogeneous degree $-\frac{1}{2}$ for $|x| \geq 1$ in $x$ and is supported outside the classical (phase-space) trajectories of quantum freely moving fragments for all possible break-ups. The latter is done by constructing observables which, unlike $A$, are $H$-bounded and have commutators with $H$ positive in parts of the phase-space region one wants to control, but - and this is the price one has to pay - in general, negative elsewhere. The positive contributions lead to the desired propagation estimates, once the negative ones are controlled. A bootstrap type procedure allows to close the argument. This approach leaves room for different constructions, which are in fact the essence of every general proof of asymptotic completeness since the first proof by Sigal and Soffer, and this is where we claim to make a significant contribution.

Our paper is organized as follows. After the preliminaries on $N$-body systems and scattering theory (Secs. 1 and 2) we essentially follow Yafaev [53] in constructing a function $g(x)$ on $X$ which grows like $|x|$, but which incorporates the full channel structure of the system (given by the asymptotics of the total potential $V(x)$ as $x \rightarrow \infty)$. Our propagation observables are derived from a modified and time-scaled Yafaev function

$$
g_{t}(x)=t^{\delta} g\left(t^{-\delta} x\right) ; \quad 0<\delta<1,
$$

or, in the Heisenberg picture, from the operator $g(t)=e^{i H t} g_{t} e^{-i H t}$. Its first derivative $\gamma(t)=\partial_{t} g(t)$ is bounded relative to $H$ and essentially increasing: $\partial_{t} \gamma(t) \geq 0$ up to terms with an integrable time decay (Sec. 3). This establishes the asymptotic observable

$$
\gamma^{+}=s-\lim _{t \rightarrow \infty} \gamma(t)=s-\lim _{t \rightarrow \infty} \frac{g(t)}{t} \geq 0,
$$

which commutes with $H$ (Sec. 4). Due to the special geometry of $g(x)$, it follows that any orbit $\psi_{t}$ in the range of $\gamma^{+}$is an outgoing scattering state:

$$
\psi_{t} \rightarrow \sum_{a} e^{-i H_{a} t} \phi_{a} \quad(t \rightarrow+\infty),
$$


where $a$ labels the channels and where $H_{a}$ is the channel Hamiltonian describing independent fragments (Sec. 5). To prove asymptotic completeness it then remains to show that the range of $\gamma^{+}$is dense in the continuous spectral subspace $\mathcal{H}_{C}$ of $H$. This is where the Mourre inequality comes in. Since $g(x)$ grows like $|x|,(0.8)$ and (0.11) lead to

$$
\theta E_{\Delta}(H) \leq \liminf _{t \rightarrow \infty} E_{\Delta}(H) \frac{g^{2}(t)}{t^{2}} E_{\Delta}(H)=E_{\Delta}(H)\left(\gamma^{+}\right)^{2} E_{\Delta}(H) .
$$

This shows that $\gamma^{+}$reduces to a strictly positive operator $\mathcal{H}_{\Delta} \mapsto \mathcal{H}_{\Delta}$. By Mourre's Theorem the subspaces of $\mathcal{H}_{\Delta}$ span $\mathcal{H}_{C}$, so that $\operatorname{Ran}\left(\gamma^{+}\right)$is indeed dense in $\mathcal{H}_{C}$.

This thumbnail sketch covers the short range case. The long range problem is qualitatively different. For reasons explained at the end of Sec. 2, we want to deal from the outset with weakly time-dependent Hamiltonians of the form $H_{t}=H+$ $W_{t}(x)$, where $W_{t}(x)$ decays like $(t+|x|)^{-\mu}$. The relation (0.11) extends easily to this more general setting, and (0.12) has a natural analogue on the range of $\gamma^{+}$(Secs. 6 and 7 ). However, $\gamma^{+}$no longer commutes with $H$, and there is no Mourre estimate for the dynamics generated by $H_{t}$ since strict energy conservation is lost. The core of the long range problem is to link the states $\psi$ with $\gamma^{+} \psi=0$ to the bound states of $H$ (Sec. 8). The difficulty here is that the spectral support of $\psi_{t}$ with respect to $H$ cannot be separated from thresholds (outside of which the Mourre inequality holds) by initial conditions. This means that the minimal distance between the fragments cannot grow proportional to $t$. An inductive procedure reduces the problem of controlling such orbits to showing that if the diameter of the system grows as $t^{\delta}$ with $\delta<1$ then the system is in a bound state. This was first done in [47] for $N \leq 4$ and in [9] for general $N$ (see also [48]). The solution of this problem also allows an effortless proof of the existence of the long range wave operators (Sec. 9). Finally, the extra propagation estimates needed in Secs. 8 and 9 are derived in Appendix C by a general method of commutator expansions, which is of independent interest (Appendix B). These estimates replace the Mourre inequality, or more precisely its consequence (0.8), in the case of time-dependent Hamiltonians.

\section{N-Body Systems}

From the standard example (0.1) we extract the three basic constituents which in this paper characterize a $N$-body system: The configuration space $X$, the lattice $L$ of channels and the intercluster potentials $I_{a}$. The resulting more general class of systems is the same as the one introduced by Agmon [1], but described from a somewhat different point of view.

Configuration Space. $X$ is an Euclidean space with a scalar product denoted by $x \cdot y$. In the example $(0.2)$ :

$$
x \cdot y=\sum_{k} m_{k}\left(x_{k} \cdot y_{k}\right)_{R^{3}} .
$$

We note that $\frac{1}{2}(\dot{x} \cdot \dot{x})=\frac{1}{2} \dot{x}^{2}$ is the classical kinetic energy and $p=\dot{x}$ the momentum conjugate to $x$. The Hamiltonian is of the form 


$$
H=\frac{1}{2} p^{2}+V(x) .
$$

In quantum mechanics this is an operator on $L^{2}(X)$, where $p=-i \nabla$ and $p^{2}=-\Delta$ have their usual form in cartesian coordinates (not the particle coordinates) of $X$.

Channels. In $X$ there is a distinguished finite lattice $L$ of subspaces $a, b, \ldots$ (channels), which is closed under intersections and which contains $\{0\}$ and $X$. In the example $(0.1)$ the channels correspond to all partitions of $(1 \ldots N)$ into subsets (clusters), e.g. if $N=4$ :

$$
\text { partition : }(12)(34) \leftrightarrow \text { channel }: a=\left\{x \in X \mid x_{1}=x_{2} ; x_{3}=x_{4}\right\} .
$$

In general we define the partial ordering of $L$ by

$$
a<b \leftrightarrow a \subset b, \quad a \neq b .
$$

For each $a \in L$ there is an orthogonal splitting

$$
X=a \oplus a^{\perp},
$$

and we write the corresponding decomposition of a vector $x \in X$ as

$$
x=x_{a}+x^{a} ; \quad x_{a} \in a, \quad x^{a} \in a^{\perp} .
$$

The example (1.3) shows that $x_{a}$ describes the CM- (center of mass) positions of the clusters and $x^{a}$ the internal configuration of each cluster in its own CM-frame (Fig. 1).

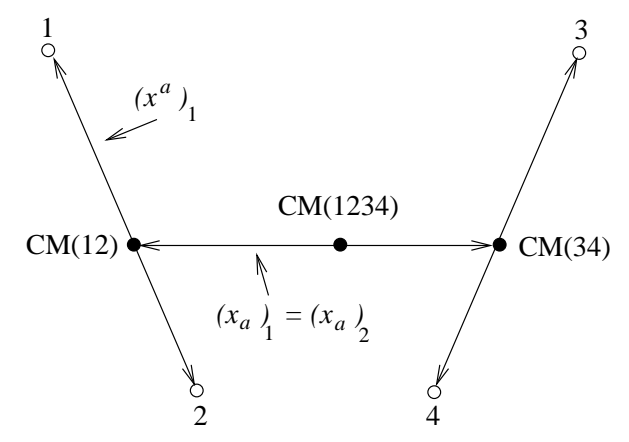

Fig. 1.

Viewed as a map of configurations in $R^{3}$, the projection $x \rightarrow x_{a}$ sends each particle into the $\mathrm{CM}$ of its cluster, while $x \rightarrow x^{a}$ translates each cluster as a whole so that its CM is relocated at the origin. We note that

$$
\frac{1}{2} p^{2}=\frac{1}{2}\left(p_{a}\right)^{2}+\frac{1}{2}\left(p^{a}\right)^{2}
$$

expresses the familiar decomposition of the kinetic energy into CM-parts and internal parts with respect to the clusters in the channel $a$. 


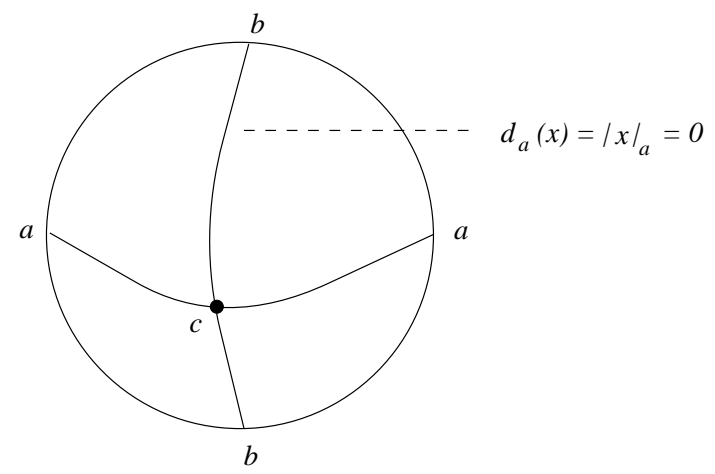

Fig. 2.

Intercluster Distance. In the example (1.3) the spatial separation of the clusters in a given configuration $x$ can be described by their distance as subsets of $R^{3}$, i.e. by

$$
d_{a}(x)=\min _{i \in(12) ; k \in(34)}\left|x_{i}-x_{k}\right| .
$$

However, it is more convenient to express the separation in terms of the geometry of $X$. Some reflection shows that

$$
d_{a}(x)=0 \Leftrightarrow x \in b, \quad b \cap a<a .
$$

Figure 2 shows the unit sphere in $X$, intersected by two channels $a, b$ with $a \cap b=$ $c<a$. This motivates our general definition of the intercluster distance:

$$
|x|_{a}:=\min _{b \cap a<a}\left|x^{b}\right| ; \quad a>\{0\} .
$$

In the case (1.3) one finds

$$
|x|_{a}=\min _{i \in(12) ; k \in(34)}\left(\frac{m_{i} m_{k}}{m_{i}+m_{k}}\right)^{1 / 2}\left|x_{i}-x_{k}\right|,
$$

which serves as well as (1.8). We note that in general, for $a>\{0\}$,

$$
a^{*}:=a \backslash \bigcup_{c<a} c=\left\{\left.x \in a|| x\right|_{a}>0\right\} .
$$

Intercluster Potentials. The basic fact about $N$-body systems is that for far separated clusters the potential depends only on the internal configuration of the clusters. To formulate this, let $a>\{0\}$ and $y \in a^{*}$. Then the translations

$$
x \rightarrow x+s y \quad(s \in R)
$$

do not affect $x^{a}$, while $|x+s y|_{a} \rightarrow \infty$ as $s \rightarrow \infty$. We therefore require that the limits

$$
V^{a}\left(x^{a}\right):=\lim _{s \rightarrow \infty} V(x+s y)
$$


exist and depend only on $x^{a}$. More precisely,

$$
V(x)=V^{a}\left(x^{a}\right)+I_{a}(x) ; \quad\left|I_{a}(x)\right| \leq f\left(|x|_{a}\right) \rightarrow 0 \text { as }|x|_{a} \rightarrow \infty .
$$

Our understanding is that the function $I_{a}(x)$ - called the intercluster potential is only defined for $|x|_{a}>R$, where $R$ is some arbitrary large constant. (For configurations where clusters are not separated the splitting of the potential into $V^{a}+I^{a}$ is artificial and should play no role in the analysis). So, if we later impose similar conditions on certain derivatives of $I_{a}(x)$, this implies only that these derivatives exist for $|x|_{a}>R$. Also, if $I_{a}(x)$ (or a derivative of $I_{a}(x)$ ) appears as an operator acting on some state $\psi \in L^{2}(X)$, this state must be supported in $|x|_{a}>R$ else the expression is not defined. On the other hand the potentials $V^{a}\left(x^{a}\right)$ are defined by (1.12) for all $x^{a} \in a^{\perp}$. To complete the definition of $V^{a}$ and $I_{a}$ we set

$$
I_{\{0\}}(x)=0 ; \quad V^{\{0\}}(x)=V(x) \quad \forall x,
$$

and since $V^{X}$ is just a constant we normalize $V(x)$ by setting

$$
V^{X}=0: I_{X}(x)=V(x) \text { for }|x|_{X}>R .
$$

In the example (1.3) we have

$$
\begin{aligned}
V^{a} & =V_{12}+V_{34} & & \left(\forall x^{a}\right) ; \\
I_{a} & =V_{13}+V_{14}+V_{23}+V_{24} & & \left(|x|_{a}>R\right) ;
\end{aligned}
$$

and in general, corresponding to $L^{2}(X)=L^{2}(a) \otimes L^{2}\left(a^{\perp}\right)$ :

$$
\begin{array}{rlrl}
H & =H_{a}+I_{a} & \left(|x|_{a}>R\right) ; \\
H_{a} & =\frac{1}{2}\left(p_{a}\right)^{2} \otimes 1+1 \otimes H^{a} & & \text { on } L^{2}(X) ; \\
H^{a} & =\frac{1}{2}\left(p^{a}\right)^{2}+V^{a} & & \text { on } L^{2}\left(a^{\perp}\right) .
\end{array}
$$

$H_{a}$ describes the dynamics of a system of non-interacting clusters and conserves $p_{a}$, $H^{a}$ describes the internal dynamics of these clusters.

Conditions on the potential. First we need some global properties of $V$ to make $H$ (and in fact all the Hamiltonians (1.14)) self-adjoint and bounded from below. Further, it is essential that the kinetic energy is bounded by the total energy in form sense:

$$
\left\langle p^{2}\right\rangle_{\psi} \leq \text { const. }\langle H+c\rangle_{\psi},
$$

where $c$ is some constant to make $H+c \geq 1$. To minimize domain considerations we make the working assumption that $V$ is a Kato-potential: For any $\alpha>0$ there exists $\beta<\infty$ such that

$$
\|V \psi\| \leq \alpha\left\|p^{2} \psi\right\|+\beta\|\psi\| \quad \forall \psi \in C_{0}^{\infty}(X) .
$$

Then $H$ is self-adjoint on $D\left(p^{2}\right)$ and satisfies $(1.15)$ [31, 39, Vol. II]. We will not reiterate this assumption in the following. The reader who is interested in $N$-body 
systems with more general (even strongly singular) potentials should consult [19]. Since our topic is scattering theory, we concentrate on the fall-off conditions for the intercluster potentials. These conditions are of the form

$$
\partial_{x}^{k} I_{a}(x)=O\left(|x|_{a}^{-\mu-|k|}\right) \quad\left(|x|_{a} \rightarrow \infty\right),
$$

in the sense of (1.13), where $k$ a multi-index. We will state the relevant values of $\mu$ and $|k|$ for each step.

Induction Principle. To summarize, we have characterized $N$-body systems by three constituents:

$$
\begin{aligned}
& \text { - a configuration space } X \\
& \text { - a lattice } L \text { of channels } \\
& \text { - a potential } V(x) \text { satisfying (1.16), (1.13), (1.17). }
\end{aligned}
$$

In this sense each Hamiltonian $H^{a}$ in (1.14) also describes a $N$-body system: its configuration space is $a^{\perp}$, its channels are the subspaces $b \cap a^{\perp}, b \geq a$, and its potential $V^{a}\left(x^{a}\right)$ is an offspring of $V(x)$ given by (1.12). It is an exercise to check that $V^{a}$ inherits all the properties we have imposed on $V$ [26]. To derive some proposition $\mathcal{P}$ from (1.18) we can therefore use induction on the lattice $L$ as follows. First, $\mathcal{P}$ is verified in the trivial case $a=X: H^{X}=0$ on $L^{2}(\{0\})=C$. Then $\mathcal{P}$ is proved for $a=\{0\}: H^{\{0\}}=H$, under the hypothesis that $\mathcal{P}$ holds for any $H^{a}$ with $a>\{0\}$. An example will be our proof of asymptotic completeness.

\section{Scattering States and Asymptotic Completeness}

Short range systems. $(\mu>1)$ Outgoing scattering states $\psi$ are characterized by the asymptotic condition

$$
\begin{aligned}
& \psi_{t}=e^{-i H t} \psi \underset{\|\|}{\longrightarrow} \sum_{a \in L} e^{-i H_{a} t} \varphi_{a} \quad(t \rightarrow+\infty) ; \\
& \varphi_{a} \in \mathcal{H}_{a}:=L^{2}(a) \otimes \mathcal{H}_{B}\left(H^{a}\right),
\end{aligned}
$$

where $\mathcal{H}_{B}\left(H^{a}\right)$ is the subspace of $L^{2}\left(a^{\perp}\right)$ spanned by the eigenvectors of $H^{a}$. Each term in the sum (2.1) represents a free motion of bound clusters in the channel $a$. For convenience we have included the bound state channel $a=\{0\}$ : if $\psi \in \mathcal{H}_{B}(H)$ then (2.1) holds trivially with $\varphi_{\{0\}}=\psi, \varphi_{a}=0$ for $a>\{0\}$. The existence of a unique scattering state $\psi$ for any given $\left\{\varphi_{a}\right\}$ is one of the earliest results in $N$-body scattering theory [22]: if $I_{a}(x)=O\left(|x|_{a}^{-\mu}\right), \mu>1$, then the wave operators

$$
\Omega_{a}^{+}=s-\lim _{t \rightarrow+\infty} e^{i H t} e^{-i H_{a} t}
$$

exist on $\mathcal{H}_{a}$, so that (2.1) holds for

$$
\psi=\sum_{a} \Omega_{a}^{+} \varphi_{a} .
$$


The wave operators are isometric from $\mathcal{H}_{a}$ to $\mathcal{H}$. Moreover, their ranges $\mathcal{H}_{a}^{+}=$ $\operatorname{Ran}\left(\Omega_{a}^{+}\right)$satisfy

$$
\mathcal{H}_{a}^{+} \perp \mathcal{H}_{b}^{+} \quad(a \neq b)
$$

expressing the fact that

$$
\lim _{t \rightarrow+\infty}\left(e^{-i H_{a} t} \varphi_{a}, e^{-i H_{b} t} \varphi_{b}\right)=0 \quad(a \neq b) .
$$

Therefore (2.4) is an orthogonal sum:

$$
\|\psi\|^{2}=\sum_{a}\left\|\Omega_{a}^{+} \varphi_{a}\right\|^{2}=\sum_{a}\left\|\varphi_{a}\right\|^{2}
$$

and the outgoing scattering states form a closed subspace

$$
\mathcal{H}^{+}=\bigoplus_{a} \mathcal{H}_{a}^{+} \subset \mathcal{H}
$$

The proofs of (2.3) and (2.6) involve only the propagation properties of free wave packets describing the center of mass motion (see e.g. [39, Vol. III]). Asymptotic completeness is the statement that

$$
\mathcal{H}^{+}=\mathcal{H},
$$

which says that any orbit $\psi_{t}$ of the system has the asymptotic form (2.1) as $t \rightarrow+\infty$, and therefore (by time reversal) also for $t \rightarrow-\infty$. The first main result we want to prove is

Theorem 2.1. (Asymptotic completeness of short range systems) (2.9) holds if for some $\mu>1$

$$
I_{a}(x)=O\left(|x|_{a}^{-\mu}\right) \quad \text { as }|x|_{a} \rightarrow \infty .
$$

Remarks. This is the result first obtained by Sigal and Soffer [44] for a somewhat smaller class of potentials, and then successively by Graf [17], Tamura [52] and Yafaev [53]. Proofs under the sole condition (2.10) and allowing singular potentials were given by Iftimovici [27], Boutet de Monvel, Georgescu and Soffer [4], and Griesemer [19]. See also [11].

Outline of the proof. An orbit $\psi_{t}$ is called asymptotically clustering if

$$
\psi_{t} \underset{\|\|}{\longrightarrow} \sum_{a} e^{-i H_{a} t} \varphi_{a} \quad(t \rightarrow+\infty)
$$

for some $\varphi_{a} \in \mathcal{H}$, i.e. without the condition (2.2). Again this is trivially true for $\psi \in \mathcal{H}_{B}(H)$. In Sec. 5 we will prove asymptotic clustering for a dense set of orbits $\psi_{t}$. Then we invoke the induction hypothesis that asymptotic completeness holds for the systems described by $H^{a}$ for all $a>\{0\}$, which is true for $a=X$. This is equivalent to saying that, for any $\varphi_{a} \in \mathcal{H}$,

$$
e^{-i H_{a} t} \varphi_{a} \underset{\|\|}{\longrightarrow} \sum_{b \geq a} e^{-i H_{b} t} \varphi_{a b} \quad(t \rightarrow \infty) ; \quad \varphi_{a b} \in L^{2}(b) \otimes \mathcal{H}_{B}\left(H^{b}\right) .
$$

Inserting (2.12) into (2.11) gives 


$$
\psi_{t} \underset{\|\|}{\longrightarrow} \sum_{b} e^{-i H_{b} t} \sum_{a \leq b} \varphi_{a b} \quad(t \rightarrow \infty),
$$

i.e. $\psi \in \mathcal{H}^{+}$. Since this holds for a dense set of $\psi$ 's and since $\mathcal{H}^{+}$is closed it follows that $\mathcal{H}^{+}=\mathcal{H}$.

Long-range systems. $(\mu \leq 1)$ For $\mu$ not too small the appropriate asymptotic condition generalizing $(2.1)$ is of the form

$$
e^{-i H t} \psi \underset{\|\|}{\longrightarrow} \sum_{a \in L} e^{-i H_{a} t-i \alpha_{a, t}\left(p_{a}\right)} \varphi_{a} \quad(t \rightarrow \infty)
$$

with $\varphi_{a} \in L^{2}(a) \otimes H_{B}\left(H^{a}\right)$ as before. In other words: only the free center-of-mass propagator of the fragments in channel $a$ is modified from

$$
e^{-\frac{i}{2} p_{a}^{2} t} \quad \text { to } \quad e^{-\frac{i}{2} p_{a}^{2} t-i \alpha_{a, t}\left(p_{a}\right)},
$$

which still conserves the momentum $p_{a}$. Here $\alpha_{a, t}\left(p_{a}\right)$ is an adiabatic phase arising from the fact that (in a classical picture) the fragments are located at

$$
x_{a}=p_{a} t\left(1+O\left(t^{-\mu}\right)\right) \quad(t \rightarrow \infty)
$$

so that

$$
I_{a}(x)=I_{a}\left(p_{a} t\right)+O\left(t^{-2 \mu}\right)
$$

provided that $\nabla I_{a}(x)=O\left(|x|_{a}^{-\mu-1}\right)$ as $|x|_{a} \rightarrow \infty$. For $2 \mu>1$ the error term in (2.16) decays integrably in time, while the leading term is of order $t^{-\mu}$ and therefore not integrable if $\mu \leq 1$. According to this classical picture the Ansatz

$$
\alpha_{a, t}\left(p_{a}\right)=\int^{t} d s I_{a}\left(p_{a} s\right)
$$

should work for $\mu>\frac{1}{2}$. The reason why we have not fully defined $\alpha_{a, t}\left(p_{a}\right)$ is twofold. First, it is clear that the modified propagator (2.15) is insensitive to a change of $\alpha_{a, t}(\cdot)$ on a null set of $a$. This allows us to restrict $p_{a}$ to the set $a^{*}(1.11)$, where $I_{a}\left(p_{a} s\right)$ indeed decays like $s^{-\mu}$. Secondly, $\alpha_{a, t}\left(p_{a}\right)$ is arbitrary within gauge transformations of the kind

$$
\alpha_{a, t}\left(p_{a}\right) \rightarrow \alpha_{a, t}\left(p_{a}\right)+f_{t}\left(p_{a}\right)
$$

if $\lim _{t \rightarrow \infty} f_{t}\left(p_{a}\right)=f_{\infty}\left(p_{a}\right)$ exists, since in (2.14) the phase $f_{\infty}\left(p_{a}\right)$ can be absorbed in $\varphi_{a}$. This is why the integrable error in (2.16) has no effect and why (2.14) is equivalent to (2.1) if $\mu>1$. A complete definition of $\alpha_{a, t}\left(p_{a}\right)$ modulo gauge transformations is therefore

$$
\alpha_{a, t}\left(p_{a}\right)=\int_{R\left|p_{a}\right|_{a}^{-1}}^{t} d s I_{a}\left(p_{a} s\right) \quad\left(p_{a} \in a^{*}\right),
$$

if for $|x|_{a}>R$

$$
\left|I_{a}(x)\right| \leq \text { const. }|x|_{a}^{-\mu} ; \quad\left|\nabla I_{a}(x)\right| \leq \text { const. }|x|_{a}^{-\mu-1} .
$$


For $a=\{0\}$ we have $p_{a}=0$ and we set $\alpha_{\{0\}, t}=0$. An important example is a system of charged particles (the Coulomb case). Then for $p_{a} \in a^{*}$

$$
I_{a}\left(p_{a} t\right)=t^{-1} \sum_{\alpha<\beta} e_{\alpha} e_{\beta}\left|\frac{p_{\alpha}}{m_{\alpha}}-\frac{p_{\beta}}{m_{\beta}}\right|^{-1},
$$

where the sum runs over all pairs of clusters in the channel $a$ with (total) charges $e_{\alpha}$, masses $m_{\alpha}$ and momenta $p_{\alpha} \in R^{3}$. A corresponding phase $\alpha_{a, t}\left(p_{a}\right)$ is simply obtained by changing the factor $t^{-1}$ to $\log (t)$. (This phase differs from (2.19) by a gauge transformation). The formulas (2.1)-(2.9) can now be transcribed to the long-range case simply by replacing

$$
H_{a} \rightarrow H_{a}+\alpha_{a, t}\left(p_{a}\right) .
$$

The proof of the existence of $\Omega_{a}^{+}$is considerably more difficult. In fact the first proofs for general $N$ without ad hoc assumptions on the decrease of bound state wave functions at infinity appeared as by-products of proofs of asymptotic completeness $([9,54])$. For the same reason we defer the proof to Sec. 9 . The second main result we prove is

Theorem 2.2. (Asymptotic completeness of long range systems) (2.9) holds if for some $\mu>\sqrt{3}-1$ and $0 \leq|k| \leq 2$

$$
\partial_{x}^{k} I_{a}(x)=O\left(|x|_{a}^{-\mu-|k|}\right) \quad \text { as }|x|_{a} \rightarrow \infty .
$$

Remark. This is the result first obtained by Dereziński [9] under somewhat stronger conditions on the potentials. The borderline $\mu=\sqrt{3}-1$ was identified by Enss for $N=3$ [15] and is further discussed in [8]. Another proof is due to Zielinski [54]. See also [11].

The proof of Theorem 2.2 will be given in Secs. 6-8. At this point we only remark that the simple induction scheme given above in the short-range case does not work in the long-range case. Proceeding inductively, we will encounter timedependent Hamiltonians similar to (2.22), acting on fibers of constant $p_{a} \in a^{*}$. This suggests an inductive proof for more general Hamiltonians of the form $H_{t}=$ $H+W_{t}(x)$ (Theorem 6.2). The special case of Theorem 2.2 is then obtained by setting $W_{t}(x) \equiv 0$ after performing the induction.

\section{Yafaev Functions and the Basic Propagation Estimate}

All our propagation observables are descendants of a time-scaled multiplication operator

$$
g_{t}(x)=t^{\delta} g\left(t^{-\delta} x\right) ; \quad 0<\delta<1,
$$

defined for $t>0$, where $g(x)$ is a positive smooth function on $X$ with the same growth as $|x|$ :

$$
0<c_{1}|x| \leq g(x) \leq c_{2}|x| \quad(|x| \geq 1) .
$$


Therefore the Mourre estimate (0.8) is equivalent to

$$
\liminf _{t \rightarrow \infty}\left\langle\frac{g_{t}^{2}}{t^{2}}\right\rangle_{t} \geq \theta,
$$

for some $\theta>0$ and for the same orbits $\psi_{t}$. Unlike $|x|$, however, $g(x)$ will be carefully adapted to the lattice of channels. In this construction we essentially follow Yafaev [53], who was motivated by a similar construction of Graf [17]. To explain the requirements we formally compute the Heisenberg derivatives

$$
\begin{aligned}
\gamma_{t}: & =D_{t} g_{t}:=i\left[H, g_{t}\right]+\partial_{t} g_{t} \\
& =\frac{1}{2}\left(\nabla g_{t} \cdot p+p \cdot \nabla g_{t}\right)+\partial_{t} g_{t} \\
D_{t}\left(\gamma_{t}-2 \partial_{t} g_{t}\right) & =p g_{t}^{\prime \prime} p-i\left[\gamma_{t}, V\right]-\frac{1}{4} \Delta^{2} g_{t}-\partial_{t}^{2} g_{t} .
\end{aligned}
$$

Here $g_{t}^{\prime \prime}(x)$ is the Hessian of $g_{t}(x)$ and (in cartesian coordinates)

$$
p g_{t}^{\prime \prime} p=\sum_{i k} p_{i} \frac{\partial^{2} g_{t}}{\partial x_{i} \partial x_{k}} p_{k} .
$$

We will call $g$ a Yafaev function if it satisfies the conditions (Y.1) and (Y.2) given below.

Condition (Y.1). $g$ is a smooth, strictly positive convex function on $X$, constant for $|x|<R^{-}$, and homogeneous of degree 1 for $|x|>R^{+}\left(0<R^{-}<R^{+}\right.$arbitrary $)$.

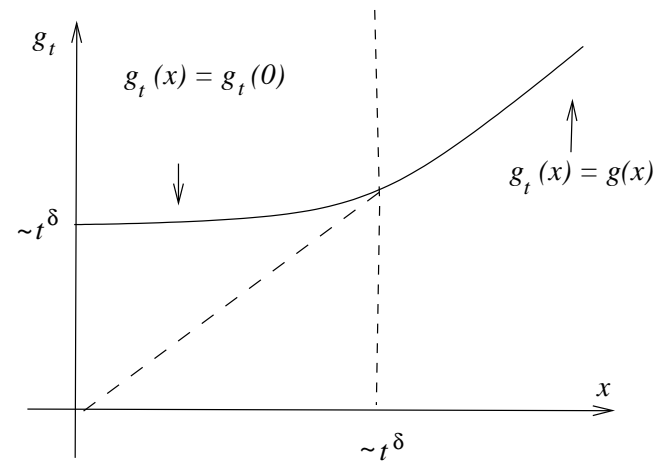

Fig. 3.

A radial section of the scaled function $g_{t}$ is shown in Fig. 3. We note that for $t \rightarrow \infty$

$$
\partial_{x}^{k} g_{t}(x)=O\left(t^{\delta(1-|k|)}\right) ; \quad \partial_{t}^{k} g_{t}(x)=O\left(t^{\delta-k}\right)
$$

uniformly in $x$. In particular, $\nabla g_{t}$ is bounded uniformly in $(x, t)$ and supported in $|x|>t^{\delta} R^{-}$, while $\partial_{t} g_{t}$ is supported in $|x|<t^{\delta} R^{+}$and bounded by const. $t^{\delta-1}$. By (1.15) $\gamma_{t}$ is defined on $D(|p|)$ and satisfies a uniform estimate

$$
\left\|\gamma_{t} \psi\right\| \leq \text { const. }\langle H+c\rangle_{\psi}^{1 / 2} \quad \forall \psi \in D(|p|) .
$$


(3.5) is understood in form sense on $D(|p|)$. The last two terms are bounded and of order

$$
\frac{1}{2} \Delta^{2} g_{t}=O\left(t^{-3 \delta}\right) ; \quad \partial_{t}^{2} g_{t}=O\left(t^{\delta-2}\right)
$$

uniformly in $x$ as $t \rightarrow \infty$. The term $p g_{t}^{\prime \prime} p$ is non-negative due to the convexity of $g$ and decays only like $t^{-\delta}$ :

$$
0 \leq p g_{t}^{\prime \prime} p \leq \text { const. } t^{-\delta}(H+c) .
$$

To treat the commutator $i\left[\gamma_{t}, V\right]$ we impose the further condition.

Condition (Y.2). For any $a>\{0\}, g(x)=g\left(x_{a}\right)$ on some cone $C_{a}$ (invariant under $x \rightarrow \lambda x)$ containing some set $\left\{x|| x^{a}\left|\leq \varepsilon_{a}\right| x \mid, \varepsilon_{a}>0\right\}$. Moreover, the cones $C_{a}$ satisfy

$$
C_{a} \cap C_{b} \subset C_{a \cap b} \quad \text { for } \quad a \cap b<a, b .
$$

Figure 4 shows a cone $C_{a}$, intersected with the compact set where $g(x)=g(0)$.

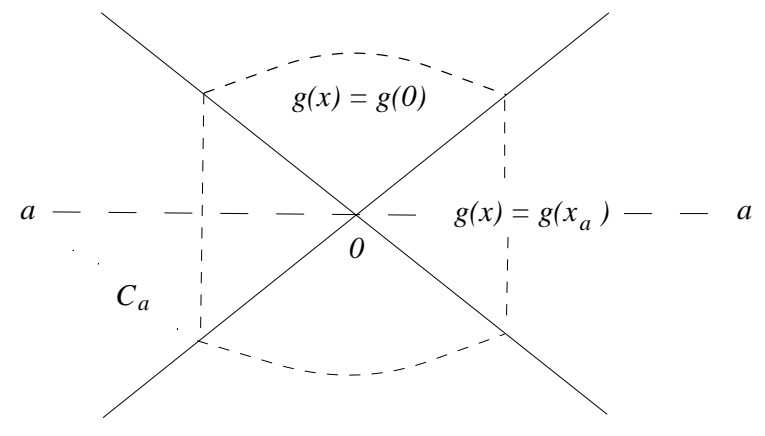

Fig. 4.

Lemma 3.1. The sets

$$
C_{a}^{*}:=C_{a} \backslash \bigcup_{b<a} C_{b}
$$

form a disjoint covering of $X$. Also

$$
\begin{gathered}
C_{b} \cap C_{a}^{*}=\phi \quad \text { if } b \cap a<a, \\
\text { and }|x|_{a} \geq|x| \cdot \min _{b>\{0\}, b \cap a<a}\left(\varepsilon_{b}\right) \quad \text { for } x \in C_{a}^{*} .
\end{gathered}
$$

Proof. The first statement follows from $C_{X}=X$ and (3.11). Let $b>\{0\}, b \cap a<a$ and $x \in C_{b} \cap C_{a}^{*}$. This excludes $b \geq a$ and $b<a$. But $b \cap a<a, b$ is equally excluded since then $x \in C_{a \cap b}$. Therefore if $x \in C_{a}^{*}$ then $\left|x^{b}\right| \geq \varepsilon_{b}|x|$ for $b \cap a<a$.

On $C_{a}^{*} \cap \operatorname{supp}\left(\nabla g_{t}\right)$ we thus have

$$
\nabla g_{t}(x) \in a ; \quad \text { and } \quad|x|_{a} \geq \lambda|x| \geq t^{\delta} \lambda R^{-}
$$


for some $\lambda>0$. If $t$ is sufficiently large we can therefore decompose $V(x)=$ $V^{a}\left(x^{a}\right)+I_{a}(x)$ in this region, which formally gives

$$
i\left[\gamma_{t}, V\right]=\nabla g_{t} \cdot \nabla I_{a}(x)
$$

since $\nabla g_{t} \cdot \nabla V^{a}=0$. In the long-range case (Theorem 2.2) we have assumed that $\nabla I_{a}(x)=O\left(|x|_{a}^{-\mu-1}\right)$, so that by $(3.14)$

$$
\left|\nabla g_{t} \cdot \nabla I_{a}\right| \leq \text { const. } t^{-\delta(\mu+1)}
$$

on each $C_{a}^{*}$. Since these sets cover $X$ this estimate holds globally on $X$ : for $t$ sufficiently large

$$
\left|\left(\psi,\left[\gamma_{t}, V\right] \psi\right)\right| \leq \text { const. } t^{-\delta(\mu+1)}\|\psi\|^{2} \quad \forall \psi
$$

In the short range case we use $\left|I_{a}(x)\right| \leq$ const. $|x|_{a}^{-\mu}$, which gives $\left|I_{a}(x)\right| \leq$ const. $t^{-\delta \mu}$ on $\operatorname{supp}\left(\nabla g_{t}\right) \cap C_{a}^{*}$ for $t$ sufficiently large. Therefore

$$
\left|\left(\psi,\left[\gamma_{t}, V\right] \psi\right)\right|=2\left|\operatorname{Im}\left(\gamma_{t} \psi, I_{a} \psi\right)\right| \leq \text { const. } t^{-\delta \mu}\langle H+c\rangle_{\psi}
$$

for $\psi \in D(|p|)$ with support in $C_{a}^{*}$. This local estimate is independent of $a$ and can be extended to all $\psi \in D(|p|)$ by using a suitable partition of unity (the sets $C_{a}^{*}$ do not form an open covering of $X$, but they can be marginally enlarged to open sets for which (3.16) prevails). Collecting these results we have in form sense for sufficiently large $t$ :

$$
\begin{aligned}
p g_{t}^{\prime \prime} p & =D_{t}\left(\gamma_{t}-2 \partial_{t} g_{t}\right)+R_{t} \\
\left|\left\langle R_{t}\right\rangle_{\psi}\right| & \leq \text { const. } t^{-\rho}\langle H+c\rangle_{\psi} \quad \forall \psi \in D(|p|) ;
\end{aligned}
$$

provided that

$$
\begin{array}{rrrr}
\text { either : } & I_{a}=O\left(|x|_{a}^{-\mu}\right) \quad \text { and } \quad \rho=\min (\delta \mu, 3 \delta, 2-\delta) \\
\text { or : } \quad \nabla I_{a}=O\left(|x|_{a}^{-\mu-1}\right) \quad \text { and } \quad \rho=\min (\delta(\mu+1), 3 \delta, 2-\delta) .
\end{array}
$$

Theorem 3.1. Suppose that (3.17) holds for some $\rho>1$. Then

$$
\int_{1}^{\infty} d t\left\langle p g_{t}^{\prime \prime} p\right\rangle_{t} \leq \text { const. }\langle H+c\rangle_{\psi} \quad \forall \psi \in D(|p|) .
$$

Proof. By (3.10) it suffices to consider the integral over an interval $t_{0}<t<\infty$ where (3.17) holds. Integrating (3.17), and using the fact that $\gamma_{t}-2 \partial_{t} g_{t}$ is bounded relative to $|p|$ uniformly in $t$, we obtain

$$
\int_{t_{0}}^{T} d t\left\langle p g_{t}^{\prime \prime} p\right\rangle_{t} \leq \text { const. }\langle H+c\rangle_{\psi}
$$

uniformly in $T$. Since the integrand is positive, the limit $T \rightarrow \infty$ exists. 
Construction of $\boldsymbol{g}(\boldsymbol{x})$. The prototype of $g(x)$ is

$$
\hat{g}(x, \sigma)=\max _{a \in L} f_{a}(x, \sigma),
$$

where $f_{\{0\}}(x, \sigma)=\sigma_{\{0\}}$ and $f_{a}(x, \sigma)=\sigma_{a}\left|x_{a}\right|$ for $a>\{0\}$. Here $\sigma=\left\{\sigma_{a}\right\}$ is a positive, decreasing function on the lattice $L$, to be adjusted in the course of the construction:

$$
\sigma_{\{0\}}>\sigma_{a}>\sigma_{b}>\sigma_{X}>0 \text { for }\{0\}<a<b<X .
$$

$\hat{g}(x, \sigma)$ is convex, constant on a compact set $K$ containing some ball $|x|<R^{-}$, and homogeneous of degree 1 in the complement of $K . \hat{g}$ has a decomposition into maximal pieces:

$$
\begin{aligned}
\hat{g}(x, \sigma) & =\sum_{a} \hat{g}_{a}(x, \sigma) \quad \text { (a.e.); } \\
\hat{g}_{a}(x, \sigma) & =f_{a}(x, \sigma) \theta\left[f_{a}(x, \sigma)-\hat{g}(x, \sigma)\right],
\end{aligned}
$$

where $\theta$ is the characteristic function of $(0, \infty)$. The piece $\hat{g}_{\{0\}}$ has support $K$ where $\hat{g}(x, \sigma)=\sigma_{\{0\}}$. The pieces $\hat{g}_{a}$ for $a>\{0\}$ have conical supports where $\hat{g}(x, \sigma)=$ $\sigma_{a}\left|x_{a}\right|$. The intersection of these cones with a sphere of radius $R^{+}$containing $K$ is shown in Fig. 5:

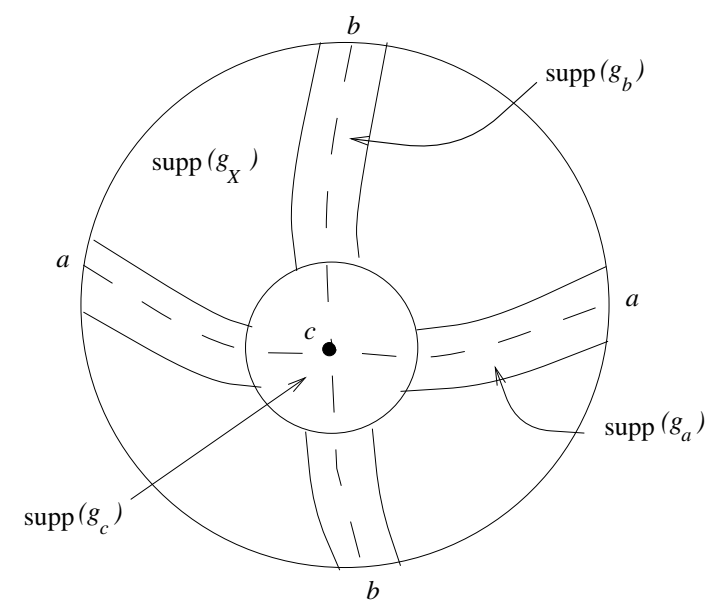

Fig. 5.

This picture corresponds to Fig. 2 and serves to explain the choice of $\sigma$. To begin with, suppose that $\sigma_{a}=\sigma_{b}=\sigma_{c}=1$. Then Fig. 4 reduces to Fig. 2 since $\left|x_{a}\right|=|x|$ exactly if $x \in a$, etc. The situation of Fig. 5 is obtained by first increasing $\sigma_{a}, \sigma_{b}$ (so that the supports of $\hat{g}_{a}, \hat{g}_{b}$ expand into strips), and then $\sigma_{c}$ sufficiently far beyond $\sigma_{a}, \sigma_{b}$ (such that supp $\hat{g}_{c}$ covers the intersection of the two strips). This corresponds to (3.11) and (3.12): in Fig. 5 the set $C_{a}^{*}$ is just the support of $\hat{g}_{a}$ where evidently

$$
|x|_{a} \geq \lambda R(\lambda>0) \text { and } \nabla g \in a
$$


except at boundary points where $\nabla g$ is discontinuous. This discontinuity is removed by a regularisation $\hat{g}(x, \sigma) \rightarrow g(x)$ which preserves convexity:

$$
g(x)=\int \hat{g}(x, \mu) \prod_{a} \tilde{\delta}\left(\mu_{a}-\sigma_{a}\right) d \mu_{a},
$$

where $0 \leq \tilde{\delta} \in C_{0}^{\infty}(R)$ is a regularized Dirac distribution with arbitrary narrow support. The same regularisation is applied to $\hat{g}_{a}(x, \sigma)$ so that

$$
g(x)=\sum_{a \in L} g_{a}(x)
$$

The analytic construction of $g$ is given in Appendix A. Here we summarize the relevant results:

Lemma 3.2. $g$ satisfies the conditions (Y.1) and (Y.2). Moreover $g$ has a decomposition (3.25) into smooth functions $g_{a}$ which have the following properties: There exists $\lambda>0$ such that

$$
\begin{aligned}
& \operatorname{supp}\left(g_{\{0\}}\right) \subset\left\{x|| x \mid<R^{+}\right\} \\
& \operatorname{supp}\left(g_{a}\right) \subset\left\{\left.x|| x\right|_{a} \geq \lambda|x| \geq \lambda R^{-}\right\} \quad \forall a>\{0\} \\
& \nabla g_{\{0\}}(x)=0 \quad \text { for }|x|<R^{-} \\
& \nabla g_{a}(x) \in b \quad \text { for } x \in C_{b}, \quad \forall b>\{0\}
\end{aligned}
$$

The functions $g_{a}$ are not convex. However, for any $a \in L$ there exists a Yafaev function $\tilde{g}$ such that the Hessians of $g_{a}$ and $\tilde{g}$ satisfy

$$
\pm g_{a}^{\prime \prime}(x) \leq \tilde{g}^{\prime \prime}(x) \quad \forall x
$$

\section{The Asymptotic Observable $\gamma^{+}$}

Corresponding to $(3.25)$ we set

$$
\begin{aligned}
& g_{t}=\sum_{a} g_{a, t} \quad: \quad g_{a, t}(x)=t^{\delta} g_{a}\left(t^{-\delta} x\right) ; \\
& \gamma_{t}=\sum_{a} \gamma_{a, t} \quad: \quad \gamma_{a, t}=D_{t} g_{a, t} .
\end{aligned}
$$

We also introduce the Heisenberg observables

$$
g(t)=e^{i H t} g_{t} e^{-i H t} ; \quad \gamma(t)=e^{i H t} \gamma_{t} e^{-i H t}=\partial_{t} g(t)
$$

and similarly for $g_{a}(t), \gamma_{a}(t)$. The operator $\gamma(t)$ is defined on $D(|p|)$, both the operators $\gamma(t)$ and $g(t)$ on the domain $D(|x|) \cap D(|p|)$, which is invariant under $\exp (-i H t)$. 
Theorem 4.1. Under the hypothesis of Theorem 3.2 the strong limits

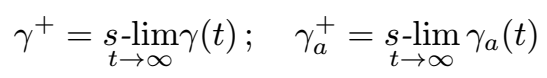

exist on $D(|p|)$ and have the following properties:

$$
\begin{aligned}
& {\left[\gamma^{+}, H\right]=0} \\
& \gamma^{+}=s-\lim _{t \rightarrow \infty} \frac{1}{t} g(t) \geq 0
\end{aligned}
$$

on $D(|x|) \cap D(|p|)$, and similarly for $\gamma_{a}^{+}$. In particular

$$
\gamma_{\{0\}}^{+}=0 ; \quad \text { i.e. } \quad \gamma^{+}=\sum_{a>\{0\}} \gamma_{a}^{+} .
$$

Moreover, $\gamma^{+}$and $\gamma_{a}^{+}$are independent of $\delta$ within the ranges allowed by the hypothesis of Theorem 3.1, since

$$
\gamma^{+}=\underset{t \rightarrow \infty}{s-\lim } e^{i H t} \frac{g}{t} e^{-i H t} \quad \text { on } D(|x|) \cap D(|p|),
$$

where $g$ is the unscaled Yafaev function $g(x)$ (and similarly for $\gamma_{a}^{+}$).

Proof. Step (1): Existence of $\gamma^{+}$. By (3.8) it suffices to prove strong convergence of $\gamma_{t}$ on the range of $(H+c)^{-2}$. First we show that

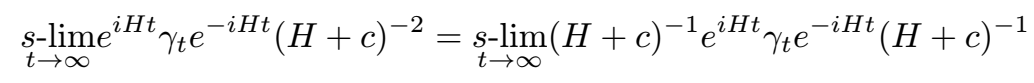

if one of these limits exists. Since $\left\|\partial_{t} g_{t}\right\|=O\left(t^{\delta-1}\right)$ we can replace $\gamma_{t}$ by $\gamma_{t}-\partial_{t} g_{t}$. Then (4.9) follows since

$$
i\left[H, \gamma_{t}-\partial_{t} g_{t}\right]=p g_{t}^{\prime \prime} p-\frac{1}{4} \Delta^{2} g_{t}-i\left[\gamma_{t}, V\right],
$$

so that by our previous estimates

$$
\left\|\left[\gamma_{t}-\partial_{t} g_{t},(H+c)^{-1}\right]\right\| \rightarrow 0 .
$$

To establish the second limit in (4.9) it suffices to prove convergence of

$$
\varphi_{t}=(c+H)^{-1} e^{i H t} \tilde{\gamma}_{t} e^{-i H t}(c+H)^{-1} \psi
$$

for all $\psi \in \mathcal{H}$, where we have chosen $\tilde{\gamma}_{t}:=\gamma_{t}-2 \partial_{t} g_{t}$. Then

$$
\partial_{t} \varphi_{t}=(H+c)^{-1} e^{i H t}\left(D_{t} \tilde{\gamma}_{t}\right) e^{-i H t}(H+c)^{-1} \psi,
$$

and we show that this is strongly integrable. By (3.5) and by our previous estimates

$$
D_{t} \tilde{\gamma}_{t}=p g_{t}^{\prime \prime} p
$$

modulo terms which give integrable contributions. So it remains to prove that

$$
u_{t}:=(H+c)^{-1} e^{i H t} p g_{t}^{\prime \prime} p e^{-i H t}(H+c)^{-1} \psi
$$


is strongly integrable over some interval $t_{0}<t<\infty$. Factorizing the positive operator $p g_{t}^{\prime \prime} p$ into $p g_{t}^{\prime \prime} p=B_{t}^{2} ; B_{t}=B_{t}^{*}$, we use the Schwarz inequality twice to estimate

$$
\begin{aligned}
\left\|\int_{t_{1}}^{t_{2}} d t u_{t}\right\|^{2} & =\sup _{\|v\|=1}\left|\int_{t_{1}}^{t_{2}} d t\left(v, u_{t}\right)\right|^{2} \\
\leq & \sup _{\|v\|=1}\left(\int_{t_{1}}^{t_{2}} d t\left\|B_{t} e^{-i H t}(H+c)^{-1} v\right\|\left\|B_{t} e^{-i H t}(H+c)^{-1} \psi\right\|\right)^{2} \\
\leq & \sup _{\|v\|=1} \int_{t_{1}}^{t_{2}} d t\left\|B_{t} e^{-i H t}(H+c)^{-1} v\right\|^{2} \\
& \times \int_{t_{1}}^{t_{2}} d t\left\|B_{t} e^{-i H t}(H+c)^{-1} \psi\right\|^{2} .
\end{aligned}
$$

By Theorem 3.1 the first factor is bounded uniformly in $t_{1}, t_{2}$, and the second factor vanishes as $t_{1,2} \rightarrow \infty$.

Step (2): Existence of $\gamma_{a}^{+}$. This is proved in the same way with two notable differences. Instead of $i\left[\gamma_{t}, V\right]$ we encounter the commutator $i\left[\gamma_{a, t}, V\right]$, formally given by

$$
i\left[\gamma_{a, t}, V\right]=\nabla g_{a, t} \cdot \nabla V .
$$

This commutator is estimated like $i\left[\gamma_{t}, V\right]$ using (3.29). Secondly, since $g_{a}$ is not convex $p g_{a, t}^{\prime \prime} p$ is not positive. Therefore we use the estimate (3.30) to split $p g_{a, t}^{\prime \prime} p$ into positive and negative parts:

$$
p g_{a, t}^{\prime \prime} p=A_{t}^{+}-A_{t}^{-} ; \quad \text { with } \quad 0 \leq A_{t}^{ \pm} \leq p \tilde{g}_{t}^{\prime \prime} p .
$$

Treating the contributions from $A_{t}^{ \pm}$separately, we then factorize $A_{t}^{ \pm}=\left(B_{t}^{ \pm}\right)^{2}$ and use the propagation estimate (3.20) for $\tilde{g}_{t}$.

Step (3): Properties of $\gamma^{+}, \gamma_{a}^{+}$. Since $\gamma^{+}$exists it follows from (4.9) that

$$
\gamma^{+}(H+c)^{-2}=(H+c)^{-1} \gamma^{+}(H+c)^{-1},
$$

i.e. $\left[\gamma^{+}, H\right]=0$ (and similarly for $\gamma_{a}^{+}$). Using that $\gamma(t)=\partial_{t} g(t)$ we have on $D(|x|) \cap D(|p|)$ :

$$
\gamma^{+}=\underset{t \rightarrow \infty}{s-\lim } \frac{1}{t} \int_{1}^{t} d s \partial_{s} g(s)=\underset{t \rightarrow \infty}{s-\lim } \frac{1}{t} g(t) \geq 0
$$

and similarly for $\gamma_{a}^{+}$. In particular

$$
\gamma_{\{0\}}^{+}=s-\lim _{t \rightarrow \infty} \frac{1}{t} g_{\{0\}}(t)=0
$$

since $\left\|g_{\{0\}}(t)\right\| \leq$ const. $t^{\delta}$. (4.8) follow from (4.6) and from the fact that

$$
\frac{1}{t}\left\|g_{t}-g\right\| \leq \text { const. } t^{\delta-1},
$$

since $g_{t}(x)-g(x)=0$ for $|x| \geq$ const. $t^{\delta}$. 
Next we discuss the connection between $\gamma^{+}$and Mourre's inequality. In addition to (4.2) we introduce the Heisenberg observable

$$
x^{2}(t)=e^{i H t} x^{2} e^{-i H t}
$$

as a form on $D(|x|) \cap D(|p|)$. This domain is not only invariant under $\exp (-i H t)$, but also (via Fourier transform) under $f(H)$ for all $f \in C_{0}^{\infty}(R)$. Let $\Delta \subset R$ be a finite interval and let $\mathcal{H}_{\Delta}$ be the corresponding spectral subspace of $H$. Since $\gamma^{+}$ commutes with $H$ and is bounded relative to $H$ it reduces to a bounded symmetric operator $\mathcal{H}_{\Delta} \rightarrow \mathcal{H}_{\Delta}$. For the following Lemma we normalize $g(x)$ such that

$$
|x| \leq g(x)
$$

Lemma 4.1. Let $\Delta \subset R$ be an open finite interval in which $H$ satisfies a Mourre estimate in form sense on $D(|x|) \cap D(|p|)$ :

$$
\liminf _{t \rightarrow \infty} f(H) \frac{x^{2}(t)}{t^{2}} f(H) \geq \theta f^{2}(H) \quad(\theta>0)
$$

for all $f \in C_{0}^{\infty}(\Delta)$. Then

$$
\gamma^{+} \geq \sqrt{\theta} \text { on } \mathcal{H}_{\Delta}
$$

in particular

$$
\mathcal{H}_{\Delta} \subset \operatorname{Ran}\left(\gamma^{+}\right) .
$$

On the other hand, if $\psi$ is an eigenvector of $H$, then

$$
\gamma^{+} \psi=0 \text {. }
$$

Proof. Since $x^{2}(t) \leq g^{2}(t)$ we obtain from (4.15) and (4.6)

$$
\theta f^{2}(H) \leq \liminf _{t \rightarrow \infty} f(H) \frac{g^{2}(t)}{t^{2}} f(H)=\left(\gamma^{+}\right)^{2} f^{2}(H)
$$

for all $f \in C_{0}^{\infty}(\Delta)$, which is equivalent to (4.16). If $\psi$ is an eigenvector of $H$ then

$$
\|\gamma(t) \psi\|=\left\|\gamma_{t} \psi\right\|
$$

and we observe that $\gamma_{t} \psi \rightarrow 0$ since $\left\|\partial_{t} g_{t}\right\| \rightarrow 0$ and $\nabla g_{t}(x)=0$ for $0 \leq|x| \leq$ $R^{-} t^{\delta}$.

We now quote the relevant parts of Mourre's Theorem in a generalized version due to Skibsted [51]. A simple proof inspired by Graf [18] is given in [19]. See also [2].

Theorem 4.2. Suppose that

$$
\begin{aligned}
\text { either }: & \lim _{|x|_{a} \rightarrow \infty}|x|_{a} I_{a}(x)=0 ; \\
\text { or }: & \lim _{|x|_{a} \rightarrow \infty}|x|_{a} \nabla I_{a}(x)=0 .
\end{aligned}
$$


Then the set $S \subset R$ given by

$$
S=\bigcup_{a \in L}\left\{\text { eigenvalues of } H^{a}\right\}
$$

is closed and countable. For any $E \in R \backslash S$ there exists an open interval $\Delta \subset R \backslash S$, $\Delta \ni E$, and a constant $\theta>0$ such that the Mourre inequality (4.15) holds.

\section{Corollary 4.1.}

$$
\mathcal{H}_{C}(H)=\overline{\operatorname{Ran}\left(\gamma^{+}\right)} ; \quad \mathcal{H}_{B}(H)=\operatorname{Ker}\left(\gamma^{+}\right)
$$

Proof. Since $S$ is countable and contains the eigenvalues of $H=H^{\{0\}}$, the spectral subspace of $H$ corresponding to $S$ is $\mathcal{H}_{B}(H)$. By Mourre's Theorem and (4.17), any state $\psi$ with compact spectral support $\subset R \backslash S$ is in $\operatorname{Ran}\left(\gamma^{+}\right)$. This implies $\mathcal{H}_{C}(H) \subset$ $\overline{\operatorname{Ran}\left(\gamma^{+}\right)}$. On the other hand it follows from (4.18) that $\mathcal{H}_{B}(H) \subset \operatorname{Ker}\left(\gamma^{+}\right)$. Since $\gamma^{+}$is selfadjoint (4.22) follows.

\section{The Short Range Case}

Theorem 5.1. If $I_{a}(x)=O\left(|x|_{a}^{-\mu}\right), \mu>1$, then the Deift-Simon wave operators

$$
\omega_{a}^{+}=\underset{t \rightarrow \infty}{s-\lim _{i}} e^{i H_{a} t} \gamma_{a, t} e^{-i H t}
$$

exist on $D(|p|)$ for $\delta$ in the range $\min (\delta \mu, 3 \delta, 2-\delta)>1$.

Proof. The proof is almost the same as the proof of the existence of $\gamma_{a}^{+}$. The modifications are as follows. Instead of (4.9) we first show that

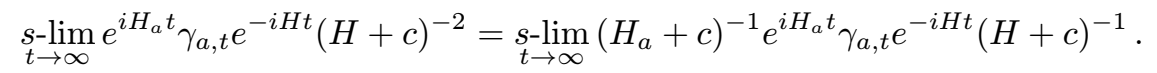

This follows from

$$
\begin{aligned}
& \left(\gamma_{a, t}-\partial_{t} g_{a, t}\right)(H+c)^{-1}-\left(H_{a}+c\right)^{-1}\left(\gamma_{a, t}-\partial_{t} g_{a, t}\right) \\
& \quad=\left(H_{a}+c\right)^{-1}\left(\left[H, \gamma_{a, t}-\partial_{t} g_{a, t}\right]-I_{a}\left(\gamma_{a, t}-\partial_{t} g_{a, t}\right)\right)(H+c)^{-1} .
\end{aligned}
$$

The extra term involving $I_{a}$ gives no contribution in the limit $t \rightarrow \infty$ since by (3.27) $\left|I_{a}(x)\right| \leq$ const. $t^{-\delta \mu}$ on $\operatorname{supp}\left(g_{a, t}\right)$. Therefore it suffices to prove convergence of

$$
\varphi_{t}=\left(H_{a}+c\right)^{-1} e^{i H_{a} t} \tilde{\gamma}_{a, t} e^{-i H t}(H+c)^{-1} \psi,
$$

where $\tilde{\gamma}_{a, t}=\gamma_{a, t}-2 \partial_{t} g_{a, t}$. Instead of (4.11) we then obtain

$$
\partial_{t} \varphi_{t}=\left(H_{a}+c\right)^{-1} e^{i H_{a} t}\left(D_{t} \tilde{\gamma}_{a, t}-i I_{a} \tilde{\gamma}_{a, t}\right) e^{-i H t}(H+c)^{-1} \psi .
$$

Here the term involving $I_{a}$ gives an integrable contribution of order $t^{-\delta \mu}$ by (3.27). The rest of the proof goes through because the propagation estimate (3.20) also holds for the dynamics generated by $H_{a}$. 
Remark. Since the roles of $H$ and $H_{a}$ in the proof of Theorem 5.1 are interchangeable, we also have

$$
\left(\omega_{a}^{+}\right)^{*}=\underset{t \rightarrow \infty}{s-\lim _{t \rightarrow} e^{i H t}} \gamma_{a, t} e^{-i H_{a} t} \quad \text { on } \quad D(|p|) .
$$

Lemma 5.1. If $I_{a}=O\left(|x|_{a}^{-\mu}\right), \mu>1$, then any orbit $\psi_{t}$ in the range of $\gamma^{+}$is asymptotically clustering:

$$
\psi_{t} \underset{\|\|}{\longrightarrow} \sum_{a>\{0\}} e^{-i H_{a} t} \varphi_{a} \quad(t \rightarrow \infty) .
$$

Proof. Let $\psi=\gamma^{+} \varphi(\varphi \in D(|p|))$. By Theorem 4.1

$$
\psi=\sum_{a>\{0\}} \gamma_{a}^{+} \varphi \underset{\|\|}{\longrightarrow} \sum_{a>\{0\}} e^{i H t} \gamma_{a, t} e^{-i H t} \varphi,
$$

and by Theorem 5.1

$$
e^{-i H t} \psi \underset{\|\|}{\longrightarrow} \sum_{a>\{0\}} e^{-i H_{a} t} e^{i H_{a} t} \gamma_{a, t} e^{-i H t} \varphi \underset{\|\|}{\longrightarrow} \sum_{a>\{0\}} e^{-i H_{a} t} \omega_{a}^{+} \varphi
$$

Lemma 5.1 together with (4.22) completes the proof of Theorem 2.1.

\section{Approach to the Long-Range Case}

We want to set up an inductive proof of asymptotic completeness for the dynamics $U_{t}: \psi \rightarrow \psi_{t}$ generated by

$$
H_{t}=H+W_{t}(x)
$$

for $0 \leq t<\infty$ with the initial condition $U_{0}=1$. We assume that

$$
\begin{gathered}
\partial_{x}^{k} I_{a}(x)=O\left(|x|_{a}^{-\mu-|k|}\right) ; \\
\left|\partial_{x, t}^{k} W_{t}(x)\right| \leq \text { const. }(1+|x|+t)^{-\mu-|k|}, \quad(0 \leq|k| \leq n)
\end{gathered}
$$

where $k$ is a multiindex and $\partial_{x, t}$ any derivative with respect to $x$ or $t$. The conditions for $\mu$ and $n$ will be given for each step. To begin with we only demand that $\mu>0$ and $n=1$. Since $W_{t}$ is bounded it is elementary to construct $U_{t} . D(H)=D\left(H_{t}\right)$ is invariant under $U_{t}$, and since

$$
\partial_{t}\left(U_{t}^{-1} H_{t} U_{t}\right)=U_{t}^{-1}\left(\partial_{t} W_{t}\right) U_{t}
$$

is norm-integrable, the limits

$$
H^{+}:=\lim _{t \rightarrow \infty} U_{t}^{-1} H_{t} U_{t}=\lim _{t \rightarrow+\infty} U_{t}^{-1} H U_{t}
$$

exist in norm sense on $D(H)$ and $H^{+}=H+B, B$ bounded. It follows that

$$
\begin{aligned}
\left(z-H^{+}\right)^{-1} & =\lim _{t \rightarrow \infty} U_{t}^{-1}(z-H)^{-1} U_{t} \\
(z-H)^{-1} & =\lim _{t \rightarrow \infty} U_{t}\left(z-H^{+}\right)^{-1} U_{t}^{-1}
\end{aligned}
$$


for $z \notin \sigma(H)=\sigma\left(H^{+}\right)$. Also $D(|p|)$ (the form domain of $H$ ) is invariant under $U_{t}$ and

$$
\left\langle p^{2}\right\rangle_{t} \leq \text { const. }\langle H+c\rangle_{\psi} .
$$

Since $D_{t} x=p$ it still follows that $D(|x|) \cap D(|p|)$ is $U_{t}$-invariant. These are the general properties of the dynamics which we have used in the short-range case. It is now straightforward to generalize the results of Secs. 3 and 4. The Heisenberg derivative $D_{t}$ refers to the evolution $U_{t}$. The expression (3.4) for $\gamma_{t}=D_{t} g_{t}$ remains unchanged, while the r.h.s. of (3.5) receives the additional term $-\nabla g_{t} \cdot \nabla W_{t}$ which is bounded in norm by const. $t^{-\delta(\mu+1)}$. As a result the basic propagation estimate (3.21) remains valid if $\delta$ is in the range

$$
\frac{1}{3}<\delta<1 ; \quad \delta(\mu+1)>1 .
$$

Under this condition the existence of

$$
\gamma^{+}=\underset{t \rightarrow \infty}{s-\lim _{t \rightarrow \infty}} \gamma(t)=\operatorname{sim}_{t \rightarrow \infty} U_{t}^{-1} \gamma_{t} U_{t} \quad \text { on } \quad D(|p|)
$$

(and similarly for $\gamma_{a}^{+}$) follows as before. We remark that in the first step of the proof (4.9) is replaced by

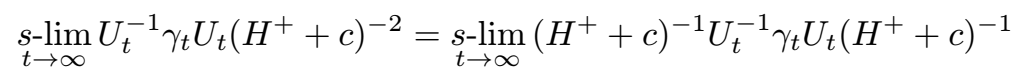

using (6.2), which leads to

$$
\left[\gamma^{+}, H^{+}\right]=0
$$

instead of $\left[\gamma^{+}, H\right]=0$ (and similarly for $\gamma_{a}^{+}$). All the other properties of $\gamma^{+}$and $\gamma_{a}^{+}$listed in Theorem 4.1 remain the same. In particular $\gamma^{+}$is independent of the choice of $\delta$ in the range (6.4). We now describe the induction proof. For each channel $a>\{0\}$ we introduce a hierarchy of time evolutions which interpolate between $U_{t}$ and the quasi-free evolution $U_{a, t}^{\infty}$ of the fragments. These evolutions have the following names and generators:

$$
\begin{aligned}
U_{t}: H_{t} & =H+W_{t}(x) ; \\
\tilde{U}_{a, t}: \tilde{H}_{a, t} & =H_{a}+W_{a, t}(x) ; \\
U_{a, t}: H_{a, t} & =H_{a}+W_{a, t}\left(p_{a} t+x^{a}\right) ; \\
U_{a, t}^{\infty}: H_{a, t}^{\infty} & =H_{a}+W_{a, t}\left(p_{a} t\right) .
\end{aligned}
$$

Here

$$
W_{a, t}(x)=\left(I_{a}(x)+W_{t}(x)\right) \chi_{a, t}(x),
$$

where $\chi_{a, t}$ is a smoothed characteristic function of the set

$$
\left\{\left.x|| x\left|\geq(1+t)^{\delta} R^{-} ; \quad\right| x\right|_{a} \geq|x|^{1-\varepsilon}\right\},
$$

with $\varepsilon>0$ arbitrary small. In particular

$$
\chi_{a, t}(\xi t)=1 \quad \text { if } \xi \in a^{*}
$$


and $t$ sufficiently large. It also follows from (3.27) that

$$
\chi_{a, t}=1 \quad \text { on } \operatorname{supp}\left(g_{a, t}\right)
$$

if $t$ is large. To compute derivatives of $\chi_{a, t}$ with respect to $t$ and $x$ one can use an explicit form of $\chi_{a, t}$ like

$$
\chi_{a, t}(x)=\theta\left((1+t)^{-\delta}|x|-R^{-}\right) \prod_{b \cap a<a} \theta\left(\left|x^{b}\right||x|^{\varepsilon-1}-1\right)
$$

where $\theta$ is a regularized step function. We note that

$$
U_{a, t}^{\infty}=e^{-i H_{a} t-i \alpha_{a, t}\left(p_{a}\right)} ; \quad \alpha_{a, t}\left(p_{a}\right)=\int_{0}^{t} d s W_{a, s}\left(p_{a} s\right) .
$$

It follows from (6.9) that on each fibre $p_{a}=\xi \in a^{*}$ this phase is equivalent to

$$
\alpha_{a, t}\left(p_{a}\right)=\int_{t_{0}\left(p_{a}\right)}^{t} d s\left(I_{a}\left(p_{a} s\right)+W_{s}\left(p_{a} s\right)\right),
$$

by a gauge transformation (2.18). Our goal is to prove the following generalization of Theorem 2.2 .

Theorem 6.1. Suppose that (6.1) holds for some $\mu>\sqrt{3}-1$ and $n=2$. Then any $\psi \in \mathcal{H}$ is an outgoing scattering state for the dynamics $U_{t}$ generated by $H_{t}$ :

$$
U_{t} \psi \underset{\|\|}{\longrightarrow} \sum_{a} e^{-i H_{a} t-i \alpha_{a}, t\left(p_{a}\right)} \varphi_{a} \quad(t \rightarrow \infty)
$$

for some $\varphi_{a} \in \mathcal{H}_{a}$. Here $\alpha_{a, t}\left(p_{a}\right)$ is given by (6.12) for $a>\{0\}$ and

$$
\alpha_{\{0\}, t}=\int_{0}^{t} d s W_{s}(0) .
$$

Outline of the Proof. The proof is given in three steps:

Step 1: Existence of the Deift-Simon wave operators

$$
\omega_{a}^{+}=\operatorname{sim}_{t \rightarrow \infty} U_{a, t}^{-1} \gamma_{a, t} U_{t} \quad \text { on } D(|p|) .
$$

This is proved in Sec. 7. It follows as in Lemma 5.1 that any $\psi \in \operatorname{Ran}\left(\gamma^{+}\right)$is asymptotically clustering: if $\psi=\gamma^{+} \varphi$ then

$$
U_{t} \psi \underset{\|\|}{\longrightarrow} \sum_{a>\{0\}} U_{a, t} \varphi_{a} ; \quad \varphi_{a}=\omega_{a}^{+} \varphi .
$$

Step 2: Induction step. We note that $H_{a, t}$ and $H_{a, t}^{\infty}$ commute with $p_{a}$. For each fiber $p_{a}=\xi \in a$ they reduce to operators acting on $L^{2}\left(a^{\perp}\right)$ given by

$$
\begin{aligned}
& H_{a, t}(\xi)=H^{a}+\frac{1}{2} \xi^{2}+W_{a, t}\left(\xi t+x^{a}\right) ; \\
& H_{a, t}^{\infty}(\xi)=H^{a}+\frac{1}{2} \xi^{2}+W_{a, t}(\xi t) .
\end{aligned}
$$


The Hamiltonian $H_{a, t}(\xi)$ has the same general form as $H_{t}$. Moreover it is not difficult to check that for any $\xi \in a^{*}$ the properties (6.1) are essentially inherited by the corresponding potential $W_{a, t}\left(\xi t+x^{a}\right)$. The only difference is that the exponents $(\mu+|k|)$ in (6.1) change into $(\mu+|k|)(1-\varepsilon)$, where $\varepsilon$ is the parameter occurring in (6.8). Since $\varepsilon$ is arbitrary small this difference is irrelevant and we ignore it. Now we invoke the induction hypothesis that asymptotic completeness in the form (6.13) holds for the dynamics $U_{a, t}(\xi)$ generated by $H_{a, t}(\xi)$ for each fiber $\xi \in a^{*}$. Integrating over the fibers gives for any $\varphi_{a} \in \mathcal{H}$ :

$$
U_{a, t} \varphi_{a} \underset{\|\|}{\longrightarrow} \sum_{b \geq a} e^{-i H_{b} t-i \alpha_{b, t}\left(p_{b}\right)} \varphi_{a b} ; \quad(t \rightarrow \infty) ; \quad \varphi_{a b} \in L^{2}(b) \otimes \mathcal{H}_{B}\left(H^{b}\right) .
$$

Inserting this into (6.16) we see that any $\psi \in \operatorname{Ran}\left(\gamma^{+}\right)$is an outgoing scattering state.

Step 3: The core of the proof is to show that (6.13) also holds for $\psi$ in the kernel of $\gamma^{+}$. In Sec. 8 we prove that $\gamma^{+} \psi=0$ implies

$$
U_{t} \psi \underset{\|\|}{\longrightarrow} e^{-i H t-i \alpha_{\{0\}, t}} \varphi ; \quad \varphi \in \mathcal{H}_{B}(H) .
$$

These three steps complete the proof of Theorem 6.1.

\section{Deift-Simon Wave Operators}

Here we use the intermediate evolution $\tilde{U}_{a, t}$ of (6.6) to factorize

$$
\begin{gathered}
\omega_{a}^{+}=w_{a}^{+} \cdot \tilde{\omega}_{a}^{+} ; \\
w_{a}^{+}=\tilde{s}_{t \rightarrow \infty} U_{a, t}^{-1} \tilde{U}_{a, t} ; \quad \tilde{\omega}_{a}^{+}=s-\lim _{t \rightarrow \infty} \tilde{U}_{a, t}^{-1} \gamma_{a, t} U_{t} .
\end{gathered}
$$

Lemma 7.1. If (6.1) holds for $\mu>0$ and $n=1$ then $\tilde{\omega}_{a}^{+}$exists on $D(|p|)$, provided that $\delta$ is taken in the range (6.4).

Proof. The proof is analogous to the proof of Theorem 5.1. We start from the equivalence

$$
\underset{t \rightarrow \infty}{s-\lim } \tilde{U}_{a, t}^{-1} \gamma_{a, t} U_{t}\left(H^{+}+c\right)^{-2}=\underset{t \rightarrow \infty}{s-\lim }\left(\tilde{H}_{a}^{+}+c\right)^{-1} \tilde{U}_{a, t}^{-1} \gamma_{a, t} U_{t}\left(H^{+}+c\right)^{-1},
$$

where $\tilde{H}_{a}^{+}:=\lim _{t \rightarrow \infty} \tilde{U}_{a, t}^{-1} \tilde{H}_{a, t} \tilde{U}_{a, t}$. This follows from (6.2) and from an identity similar to (5.2), where the term $I_{a}\left(\gamma_{a, t}-\partial_{t} g_{a t}\right)$ is now replaced by

$$
\left(H_{t}-\tilde{H}_{a, t}\right)\left(\gamma_{a, t}-\partial_{t} g_{a, t}\right)=\left(I_{a}+W_{t}\right)\left(1-\chi_{a, t}\right)\left(\gamma_{a, t}-\partial_{t} g_{a, t}\right) .
$$

This term vanishes exactly for $t$ sufficiently large by (6.10). Then we have to prove convergence of

$$
\varphi_{t}=\left(\tilde{H}_{a}^{+}+c\right) \tilde{U}_{a, t}^{-1} \tilde{\gamma}_{a, t} U_{t}\left(H^{+}+c\right)^{-1} \psi,
$$

where $\tilde{\gamma}_{a, t}=\gamma_{a, t}-2 \partial_{t} g_{a, t}$. Instead of (5.3) we obtain

$$
\partial_{t} \varphi_{t}=\left(\tilde{H}_{a}^{+}+c\right)^{-1} \tilde{U}_{a, t}^{-1}\left(D_{t} \tilde{\gamma}_{a, t}\right) U_{t}\left(H^{+}+c\right)^{-1} \psi
$$


since the term $\left(H_{t}-\tilde{H}_{a, t}\right) \tilde{\gamma}_{a, t}$ vanishes again by (6.10). Strong integrability of $\partial_{t} \varphi_{t}$ follows as before from the propagation estimate (3.20), which also holds for the evolution $\tilde{U}_{a, t}$.

Lemma 7.2. If (6.1) holds for $\mu>1 / 2$ and $n=2$ then $w_{a}^{+}$exists on $\mathcal{H}$ provided that $\delta$ is taken in the range

$$
\frac{1}{3}<\delta<1 ; \quad \delta(\mu+1)>\frac{3}{2} ; \quad \delta(\mu+2)>2 .
$$

Proof. Using Cook's argument we want to show that

$$
\partial_{t} U_{a, t}^{-1} \tilde{U}_{a, t} \psi=-i U_{a, t}^{-1}\left[W_{a, t}(x)-W_{a, t}\left(p_{a} t+x^{a}\right)\right] \tilde{U}_{a, t} \psi
$$

is integrable for a dense set of $\psi$ 's. The middle factor can be expressed as

$$
\begin{aligned}
{[\cdots]=} & \int_{0}^{1} d s \nabla_{a} W_{a, t}\left(s x_{a}+(1-s) p_{a} t+x^{a}\right) \cdot\left(x_{a}-p_{a} t\right) \\
& +\frac{i t}{2} \int_{0}^{1} d s \Delta_{a} W_{a, t}\left(s x_{a}+(1-s) p_{a} t+x^{a}\right) .
\end{aligned}
$$

This identity comes from evaluating

$$
f(x)-f(p t)=\int_{0}^{1} d s \frac{d}{d s} f(p t+s(x-p t)) .
$$

Representing $f(x)$ by a Fourier integral, say for $f \in \mathcal{S}\left(R^{n}\right)$, it suffices to consider the case $f(x)=\exp (i k \cdot x)$. Then

$$
e^{i k \cdot(p t+s(x-p t))}=e^{i t k \cdot p} e^{i s k \cdot(x-p t)} e^{-\frac{i}{2} s t k^{2}}
$$

by the Campbell-Hausdorff formula. Now the $s$-derivative is computed and inserted into the Fourier integral. This proves (7.4) for $W_{t}(\cdot) \in \mathcal{S}\left(R^{n}\right)$, and our bounds for $\nabla W_{t}$ and $\Delta W_{t}$ allow it to extend the result by a limiting argument. From (6.7) and (6.11) it follows that

$$
\left\|\Delta_{a} W_{a, t}\right\|=\sup _{x}\left|\Delta_{a} W_{a, t}(x)\right| \leq \text { const. }(1+t)^{-\delta(\mu+2)(1-\varepsilon)} .
$$

Since $\varepsilon$ is arbitrary small we drop the factor $(1-\varepsilon)$. The second term in (7.4) is therefore norm-bounded by const. $t^{1-\delta(\mu+2)}$, which is integrable since $\delta(\mu+2)>2$. Similarly, the contribution of the first term is bounded by

$$
\text { const. } t^{-\delta(\mu+1)}\left\langle\left(x_{a}-p_{a} t\right)^{2}\right\rangle_{t}^{1 / 2}
$$

where $\langle\cdots\rangle_{t}$ is taken for the evolution $\psi_{t}=\tilde{U}_{a, t} \psi$. Let $\tilde{D}_{t}$ be the corresponding Heisenberg derivative. Then

$$
\begin{aligned}
\tilde{D}_{t}\left(x_{a}-p_{a} t\right) & =i\left[\tilde{H}_{a, t}, x_{a}-p_{a} t\right]-p_{a}=i\left[W_{a, t}(x), x_{a}-p_{a} t\right] \\
& =t \nabla_{a} W_{a, t}(x)=O\left(t^{1-\delta(\mu+1)}\right)
\end{aligned}
$$


uniformly in $x$. Therefore

$$
\begin{aligned}
\left|\partial_{t}\left\langle\left(x_{a}-p_{a} t\right)^{2}\right\rangle_{t}\right| & \leq \text { const. } t^{1-\delta(\mu+1)}\left\langle\left(x_{a}-p_{a} t\right)^{2}\right\rangle_{t}^{1 / 2}, \quad \text { or } \\
\left|\partial_{t}\left\langle\left(x_{a}-p_{a} t\right)^{2}\right\rangle^{1 / 2}\right| & \leq \text { const. } t^{1-\delta(\mu+1)}
\end{aligned}
$$

Taking $\psi \in D(|x|) \cap D(|p|)$ this gives

$$
\left\langle\left(x_{a}-p_{a} t\right)^{2}\right\rangle_{t}^{1 / 2} \leq \text { const. } t^{2-\delta(\mu+1)} .
$$

Together with (7.5) we see that the contribution of the first term in (7.4) is integrable if $2 \delta(\mu+1)>3$.

We remark that this proof is the only instance where we use the second derivatives of $I_{a}(x)$ and $W_{t}(x)$ with respect to $x$. As a result we have:

Theorem 7.1. If (6.1) holds for $\mu>1 / 2$ and $n=2$ then the Deift-Simon wave operators

$$
\omega_{a}^{+}=s-\lim _{t \rightarrow \infty} U_{a, t}^{-1} \gamma_{a, t} U_{t}
$$

exist on $D(|p|)$ provided that $\delta$ is taken in the range

$$
\frac{1}{3}<\delta<1 ; \quad \delta(\mu+1)>32 ; \quad \delta(\mu+2)>2 .
$$

Remark. This Theorem and its proof using the factorization (7.1) are due to Dereziński and Gérard [10].

Corollary 7.1. The adjoints of $\omega_{a}^{+}$exist as strong limits

$$
\left(\omega_{a}^{+}\right)^{*}=\underset{t \rightarrow \infty}{s-\lim _{t}} U_{t}^{-1} \gamma_{a, t} U_{a, t} \quad \text { on } D(|p|) .
$$

Proof. The roles of $U_{t}, \tilde{U}_{a, t}$ in the proof of Lemma 7.1 and of $U_{a, t}, \tilde{U}_{a, t}$ in the proof of Lemma 7.2 are interchangeable.

\section{Propagation on $\operatorname{Ker}\left(\gamma^{+}\right)$}

Theorem 8.1. Let $\gamma^{+} \psi=0$ and suppose that (6.1) holds for $\mu>\sqrt{3}-1$ and $n=1$. Then

$$
U_{t} \psi \underset{\|\|}{\longrightarrow} e^{-i H t-i \int_{0}^{t} d s W_{s}(0)} \varphi \quad(t \rightarrow \infty) ; \quad \varphi \in \mathcal{H}_{B}(H) .
$$

Remarks. The scaling parameter $\delta$ does not appear in this theorem. However, we will need the propagation estimate (3.20) and the representation of $\gamma^{+}$as the strong limit (4.4), i.e. some choice of $\delta$ in the range

$$
\frac{1}{3}<\delta<1 ; \quad \delta(\mu+1)>1,
$$


in which $\gamma^{+}$is independent of $\delta$ (Theorem 4.1). For the proof we also need the additional conditions

$$
\delta<\mu ; \quad \delta(\mu+2)>2 .
$$

(8.2) and (8.3) can both be satisfied if $\mu(\mu+2)>2$, i.e. if $\mu>\sqrt{3}-1$. The proof of Theorem 8.1 is given in 3 steps (Lemmas $8.1-8.3$ below), where we use the notation and the results of Appendix C. We also recall

$$
U_{\{0\}, t}^{\infty}:=e^{-i H t-i \int_{0}^{t} d s W_{s}(0)} .
$$

Lemma 8.1. Let $\delta<\delta^{\prime}<\mu$ and $\theta>0$. Then

$$
\omega_{\{0\}}^{+}=\underset{t \rightarrow \infty}{s-\lim }\left(U_{\{0\}, t}^{\infty}\right)^{-1} \chi\left(t^{-\delta^{\prime}} g_{t} \leq \theta\right) U_{t}
$$

exists on $\mathcal{H}$.

Lemma 8.2. If $\gamma^{+} \psi=0$ and $\delta>\frac{2}{3}$ then

$$
\omega_{\{0\}}^{+} \psi=\lim _{t \rightarrow \infty}\left(U_{\{0\}, t}^{\infty}\right)^{-1} U_{t} \psi
$$

Lemma 8.3. If $\gamma^{+} \psi=0$ and

$$
U_{t} \psi \underset{\|\|}{\longrightarrow} e^{-i H t-i \alpha(t)} \varphi \quad(t \rightarrow \infty)
$$

for some real phase $\alpha(t)$, then $\varphi \in \mathcal{H}_{B}(H)$.

Proof of Lemma 8.1. Since

$$
\left\|\left[(H+c)^{-1}, \chi\left(t^{-\delta^{\prime}} g_{t} \leq \theta\right)\right]\right\|=O\left(t^{-\delta^{\prime}}\right)
$$

it suffices to prove strong convergence of

$$
\phi_{t}=\left(U_{\{0\}, t}^{\infty}\right)^{-1}(H+c)^{-1} \chi\left(t^{-\delta^{\prime}} g_{t} \leq \theta\right)(H+c)^{-1} U_{t} \psi .
$$

Proceeding as in the proof of Theorem 4.1 we estimate

$$
\begin{aligned}
\partial_{t} \phi_{t}= & \left(U_{\{0\}, t}^{\infty}\right)^{-1}(H+c)^{-1}\left(D_{t} \chi\right)(H+c)^{-1} U_{t} \\
& +\left(U_{\{0\}, t}^{\infty}\right)^{-1}(H+c)^{-1}\left[W_{t}(0)-W_{t}(x)\right] \chi(H+c)^{-1} U_{t},
\end{aligned}
$$

where $D_{t}$ is the Heisenberg derivative for $U_{t}$. The first term is strongly integrable by Theorem C.2 and by the argument given in (4.12). The same is true for the second term since $|x| \leq$ const. $t^{\delta^{\prime}}$ on $\operatorname{supp}\left(\chi\left(t^{-\delta^{\prime}} g_{t} \leq \theta\right)\right.$. Using (6.1) we thus obtain

$$
\left\|\left(W_{t}(0)-W_{t}(x)\right) \chi\left(t^{-\delta^{\prime}} g_{t} \leq \theta\right)\right\|=O\left(t^{\delta^{\prime}-\mu-1}\right),
$$

which is integrable since $\delta^{\prime}<\mu$. 
Proof of Lemma 8.2. Since $\left[\gamma^{+}, H^{+}\right]=0$ it suffices to prove (8.6) for $\psi \in D(H)$. By Lemma 8.1 we need only show that for $\psi_{t}=U_{t} \psi$

$$
\lim _{t \rightarrow \infty} \chi\left(t^{-\delta^{\prime}} g_{t} \geq \theta\right) \psi_{t}=0
$$

for $\theta>0$ or, by (C.5), that

$$
\lim _{t \rightarrow \infty} \chi\left(t^{1-\delta^{\prime}} \Gamma_{t} \geq \theta_{1}\right) \psi_{t}=0
$$

for $\theta_{1}>0$. Since $\left\|\partial_{t} g_{t}\right\| \rightarrow 0, \gamma^{+} \psi=0$ implies that $\Gamma_{t} \psi_{t} \rightarrow 0$ for $t \rightarrow \infty$, and therefore

$$
\lim _{s \rightarrow \infty} \chi\left(b \Gamma_{s} \geq \theta_{1}\right) \psi_{s}=0
$$

for any $b>0$. Using this and Lemma C.1 we can estimate

$$
\begin{aligned}
\left\langle\chi\left(b \Gamma_{s} \geq \theta_{1}\right)\right\rangle_{s} & =-\int_{s}^{\infty} d t\left\langle D_{t} \chi\left(b \Gamma_{s} \geq \theta_{1}\right)\right\rangle_{t} \\
& \leq \text { const. }\left(b^{3} s^{1-3 \delta}+b s^{1-\delta(\mu+1)}\right)\|\psi\|_{H}^{2} .
\end{aligned}
$$

Setting now $s=t, b=t^{1-\delta^{\prime}}$ we obtain

$$
\left\langle\chi\left(t^{1-\delta^{\prime}} \Gamma_{t} \geq \theta_{1}\right)\right\rangle_{t}=O\left(t^{4-6 \delta}\right)+O\left(t^{2-\delta(\mu+2)}\right)
$$

which vanishes as $t \rightarrow \infty$. Replacing $\chi$ by $\chi^{2}$ we arrive at (8.8).

Proof of Lemma 8.3. Again we can assume that $\psi \in D\left(H^{+}\right)$. Then (8.6) and (6.2) imply $\varphi \in D(H)$ and

$$
0=\lim _{t \rightarrow \infty}\left(\psi, U_{t}^{-1} \gamma_{t} U_{t} \psi\right)=\lim _{t \rightarrow \infty}\left(\varphi, e^{i H t} \gamma_{t} e^{-i H t} \varphi\right)=\left(\varphi, \gamma_{H}^{+} \varphi\right)
$$

where $\gamma_{H}^{+}$is the asymptotic observable $\gamma^{+}$for the time evolution generated by $H$. Since $\gamma_{H}^{+} \geq 0$ this implies $\gamma_{H}^{+} \varphi=0$ and therefore $\varphi \in \mathcal{H}_{B}(H)$ by (4.22).

\section{Long-Range Wave Operators}

In this section, we prove the existence of the wave operators in the long-range case for the dynamics $U_{t}$ generated by $H_{t}$.

Theorem 9.1. Suppose that (6.1) holds for some $\mu>\sqrt{3}-1$ and $n=2$. Then the wave operators

$$
\Omega_{a}^{+}=s-\lim _{t \rightarrow \infty} U_{t}^{-1} U_{a, t}^{\infty}
$$

exist on $\mathcal{H}_{a}$ for all $a \in L$ and have mutually orthogonal ranges

$$
\mathcal{H}_{a}^{+} \perp \mathcal{H}_{b}^{+} \quad(a \neq b) .
$$

The proof of this theorem uses the results of Secs. 7 and 8. The first step is to prove the special case $a=\{0\}$ : 
Lemma 9.1. Under the hypothesis of Theorem 9.1 the wave operator

$$
\Omega_{\{0\}}^{+}=\underset{t \rightarrow \infty}{s-\lim _{t}} U_{t}^{-1} U_{\{0\}, t}^{\infty}
$$

exists on $\mathcal{H}_{B}(H)$.

Proof. Let $\psi$ be an eigenvector of $H$. Since the time evolution $\psi_{t}=U_{\{0\}, t}^{\infty} \psi$ affects only the phase of $\psi$ it follows directly that

$$
\lim _{t \rightarrow \infty}\left\|\chi\left(t^{-\delta^{\prime}} g_{t} \geq \theta\right) \psi_{t}\right\|=0
$$

for $\delta^{\prime}>\delta$. Therefore it suffices to establish strong convergence of

$$
U_{t}^{-1} \chi\left(t^{-\delta^{\prime}} g_{t} \leq \theta\right) U_{\{0\}, t}^{\infty} .
$$

This is proven like Lemma 8.1, with the roles of the two propagators interchanged.

Our goal is to prove convergence of (9.1) for $a>\{0\}$ on the states of the form

$$
\psi(x)=\varphi\left(x_{a}\right) u\left(x^{a}\right) ; \quad \hat{\varphi} \in C_{0}^{\infty}\left(a^{*}\right) ; \quad H^{a} u=\lambda u,
$$

where $\hat{\varphi}$ is the Fourier transform of $\varphi$. These states span a dense set in $\mathcal{H}_{a}$.

Lemma 9.2. Under the hypothesis of Theorem 9.1 the limits

$$
\lim _{t \rightarrow \infty} U_{t}^{-1} \gamma_{a, t} U_{a, t}^{\infty} \psi
$$

exist for $a>\{0\}$ and any $\psi$ of the form (9.4).

Proof. Evidently we can write $\psi=\left(H_{a}+i\right)^{-1} \tilde{\psi}$ with $\tilde{\psi}$ of the form (9.4). Since $H_{a}$ commutes with $U_{a, t}^{\infty}$ we can factorize

$U_{t}^{-1} \gamma_{a, t} U_{a, t}^{\infty} \psi=\left(U_{t}^{-1} \gamma_{a, t} U_{a, t}\left(H_{a}^{+}+i\right)^{-1}\right)\left(\left(H_{a}^{+}+i\right) U_{a, t}^{-1}\left(H_{a}+i\right)^{-1} U_{a, t}\right)\left(U_{a, t}^{-1} U_{a, t}^{\infty} \tilde{\psi}\right)$.

All three factors are bounded uniformly in $t$. The first factor converges strongly by Corollary 7.1. The second factor converges to 1 in norm by (6.2), applied to $H_{a}$ in place of $H$. Since $p_{a}$ commutes with $U_{a, t}^{-1} U_{a, t}^{\infty}$ it suffices to prove convergence of the last factor in $\mathcal{H}^{a}$ for each fiber $p_{a}=\xi \in a^{*}$. Recalling the generators (6.17) this follows from Lemma 9.1, applied to $H^{a}+W_{a, t}\left(\xi t+x^{a}\right)$ in place of $H_{t}$.

Lemma 9.3. Suppose that (6.1) holds for some $\mu>0$ and $n=2$. Then, for $a>\{0\}$ and any fixed $\psi$ of the form (9.4), we can choose the Yafaev function $g$ such that

$$
\lim _{t \rightarrow \infty}\left(U_{a, t}^{\infty}\right)^{-1} \gamma_{a, t} U_{a, t}^{\infty} \psi=\sigma_{a}\left|p_{a}\right| \psi, \quad\left(\sigma_{a}>0\right)
$$


As a preparation for the proof we discuss the asymptotic form of the quasi-free evolution in $L^{2}\left(R^{n}\right)$ :

$$
\varphi_{t}=e^{-\frac{i}{2} t p^{2}} \varphi_{t}^{0} ; \quad \varphi_{t}^{0}=e^{-i \alpha_{t}(p)} \varphi
$$

for $\hat{\varphi} \in C_{0}^{\infty}\left(R^{n} \backslash\{0\}\right)$. Here

$$
\alpha_{t}(p)=\int_{0}^{t} d s W_{s}(p s),
$$

and $W_{t}(x)$ is assumed to satisfy (6.1) with $\mu>0$ and $n=2$. Then

$$
\varphi_{t}(x)=(2 \pi i t)^{-n / 2} \exp \left(\frac{i x^{2}}{2 t}\right) \int d y \exp \left(-\frac{i x \cdot y}{t}\right) \exp \left(\frac{i y^{2}}{2 t}\right) \varphi_{t}^{0}(y) .
$$

The lowest order Taylor expansion of the last exponential leads to

$$
\begin{gathered}
\varphi_{t}(x)=\tilde{\varphi}_{t}(x)+R_{t}(x) ; \quad \tilde{\varphi}_{t}(x)=(i t)^{-n / 2} \exp \left(\frac{i x^{2}}{2 t}\right) \widehat{\varphi_{t}^{0}}\left(\frac{x}{t}\right) ; \\
\left\|R_{t}(\cdot)\right\|_{2} \leq \frac{1}{t}\left\|\Delta\left(\exp \left(-i \alpha_{t}(\cdot)\right) \hat{\varphi}_{0}(\cdot)\right)\right\|_{2}=O\left(t^{-\mu}\right) .
\end{gathered}
$$

$\tilde{\varphi}_{t}$ is the leading term for $t \rightarrow \infty$. The bound for $R_{t}$ is obtained by estimating $\nabla \alpha_{t}(\cdot)$ and $\Delta \alpha_{t}(\cdot)$ using $(6.1)$. As a result we have

$$
\lim _{t \rightarrow \infty}\left\|\varphi_{t}-\tilde{\varphi}_{t}\right\|=0 \quad \text { and } \quad \lim _{t \rightarrow \infty}\left\|p_{k} \varphi_{t}-\left(\frac{x_{k}}{t}\right) \tilde{\varphi}_{t}\right\|=0 .
$$

The second limit follows from the first if $\varphi$ is replaced by $p_{k} \varphi$.

Proof of Lemma 9.3. Given $\psi$ of the form (9.4) we can choose the Yafaev function $g$ such that for some $\varepsilon>0$

$$
g_{a}(x)=\sigma_{a}\left|x_{a}\right| \quad \text { if } \quad x \in \operatorname{supp}(\hat{\varphi}) \quad \text { and } \quad\left|x^{a}\right| \leq \varepsilon
$$

(cf. Lemma A.3). Then

$$
g_{a, t}(x)=\sigma_{a}\left|x_{a}\right| \quad \text { if } \quad \frac{x}{t} \in \operatorname{supp}(\hat{\varphi}) \quad \text { and } \quad\left|x^{a}\right| \leq \varepsilon t
$$

for $t>1$. Since

$$
\gamma_{a, t}=\nabla g_{a, t} \cdot p+O\left(t^{-\delta}\right)
$$

we have

$$
\gamma_{a, t} U_{a, t}^{\infty} \psi \underset{\|\|}{\longrightarrow} \nabla g_{a, t} \cdot\left(p_{a} \varphi_{t} \otimes e^{-i \lambda t} u\right)+\nabla g_{a, t} \cdot\left(\varphi_{t} \otimes e^{-i \lambda t} p^{a} u\right)
$$

where $\varphi_{t}$ is the quasi-free evolution of $\varphi$ in $L^{2}(a)$. Using (9.8) we obtain

$$
\gamma_{a, t} U_{a, t}^{\infty} \psi \underset{\|\|}{\longrightarrow} \nabla g_{a, t} \cdot\left(\frac{x_{a}}{t} \tilde{\varphi}_{t} \otimes e^{-i \lambda t} u\right)+\nabla g_{a, t} \cdot\left(\tilde{\varphi}_{t} \otimes e^{-i \lambda t} p^{a} u\right) .
$$


The part of this wave function in the region $\left|x^{a}\right|>\varepsilon t$ vanishes in norm as $t \rightarrow \infty$ since $u$ and $p^{a} u$ are in $L^{2}\left(a^{\perp}\right)$. In the complementary region we have $\nabla g_{a, t}=$ $\sigma_{a} x_{a}\left|x_{a}\right|^{-1} \in a$, so that the last term of (9.9) gives no contribution. Therefore

$$
\gamma_{a, t} U_{a, t}^{\infty} \psi \underset{\|\|}{\longrightarrow} \sigma_{a} \frac{\left|x_{a}\right|}{t}\left(\tilde{\varphi}_{t} \otimes e^{-i \lambda t} u\right) \underset{\|\|}{\longrightarrow} \sigma_{a}\left|p_{a}\right| U_{a, t}^{\infty} \psi
$$

Proof of Theorem 9.1. By (9.5) and (9.6)

$$
\lim _{t \rightarrow \infty} U_{t}^{-1} \gamma_{a, t} U_{a, t}^{\infty} \psi=\lim _{t \rightarrow \infty} U_{t}^{-1} U_{a, t}^{\infty} \sigma_{a}\left|p_{a}\right| \psi=\Omega_{a}^{+} \sigma_{a}\left|p_{a}\right| \psi
$$

exists. Since $\sigma_{a}\left|p_{a}\right|$ maps the class (9.4) onto itself, $\Omega_{a}^{+} \psi$ exists for all $\psi$ in this class. (9.2) follows from

$$
\lim _{t \rightarrow \infty}\left(U_{a, t}^{\infty} \psi_{a}, U_{b, t}^{\infty} \psi_{b}\right)=0
$$

for $a \neq b$ and $\psi_{a}, \psi_{b}$ of the form (9.4). This is readily obtained by using the asymptotic form (9.7).

\section{Acknowledgments}

This work was supported by the Swiss National Fund (WH) and by the NSERC under Grant NA7901 (IMS). Each author enjoyed the hospitality of his co-author's home institution during extended periods. We thank A. Soffer and G. M. Graf for many years of fruitful collaboration on scattering theory. We are also indebted to the referee for his thorough review, which in particular prevented a mistake in the proof of Theorem C.2.

\section{Appendix A. The Yafaev Construction}

In this appendix we describe the analytic construction of $g(x)$ as outlined in Sec. 3. For $a>\{0\}$ and $\sigma$ given in accordance with (3.22) we define the cones

$$
\begin{aligned}
& C_{a}(\sigma)=\left\{x\left|\sigma_{a}\right| x_{a}\left|>\sigma_{b}\right| x_{b} \mid \quad \forall b>a\right\} \\
& U_{a}(\sigma)=\left\{x\left|\sigma_{a}\right| x_{a}\left|>\sigma_{X}\right| x \mid\right\} ; \\
& V_{a}(\sigma)=\left\{x\left|\sigma_{a}\right| x_{a}\left|>\sigma_{b}\right| x \mid \quad \forall b>a\right\} .
\end{aligned}
$$

Evidently $V_{a}(\sigma) \subset C_{a}(\sigma) \subset U_{a}(\sigma)$ and

$$
x \in U_{a} \Leftrightarrow\left(x^{a}\right)^{2}<\left(1-\frac{\sigma_{X}^{2}}{\sigma_{a}^{2}}\right) x^{2} ; \quad x \in V_{a} \Leftrightarrow\left(x^{a}\right)^{2}<\left(1-\frac{\sigma_{b}^{2}}{\sigma_{a}^{2}}\right) x^{2} \quad \forall b>a .
$$

We note that $V_{a}(\sigma)$ and hence $C_{a}(\sigma)$ contains the cone

$$
\left(x^{a}\right)^{2}<x^{2} \cdot \min _{b>a}\left(1-\frac{\sigma_{b}^{2}}{\sigma_{a}^{2}}\right) .
$$

Lemma A.1. There exists a choice of $\sigma_{a}, a>\{0\}$ such that for $a \cap b<a, b$

$$
U_{a}(\sigma) \cap U_{b}(\sigma) \subset V_{a \cap b}(\sigma) .
$$


Proof. Let $0<\varepsilon<1$ and $|a|=\operatorname{dim}(a)$. We define $\sigma_{a}$ by

$$
1-\frac{1}{\sigma_{a}^{2}}=\varepsilon^{2|a|}
$$

Then, for $b>a$,

$$
\varepsilon^{2|a|}>\left(1-\frac{\sigma_{b}^{2}}{\sigma_{a}^{2}}\right)=\frac{\varepsilon^{2|a|}-\varepsilon^{2|b|}}{1-\varepsilon^{2|b|}}>\varepsilon^{2|a|}\left(1-\varepsilon^{2}\right)
$$

since $|b| \geq|a|+1$. Now we use that

$$
\left|x^{a \cap b}\right| \leq M \cdot \max \left(\left|x^{a}\right|,\left|x^{b}\right|\right)
$$

for all $x, a, b$, where $M$ is a constant depending only on the lattice $L$ (Both sides are seminorms on $X$, and $\left|x^{a}\right|=\left|x^{b}\right|=0$ implies $x \in a \cap b$, i.e. $\left.\left|x^{a \cap b}\right|=0\right)$. Let $a \cap b=c<a, b$ and $x \in U_{a} \cap U_{b}$. Then

$$
\left|x^{c}\right|^{2} \leq M^{2} \cdot \max \left(\varepsilon^{2|a|}, \varepsilon^{2|b|}\right) x^{2} \leq M^{2} \varepsilon^{2(|c|+1)} x^{2} .
$$

It follows that $x \in V_{c}(\sigma)$ if $M^{2} \varepsilon^{2(|c|+1)} \leq \varepsilon^{2|c|}\left(1-\varepsilon^{2}\right)$, i.e. if $\varepsilon^{2} \leq\left(1+M^{2}\right)^{-1}$.

Lemma A.1 and (A.1) show that the family of cones $C_{a}(\sigma)$ has all the properties required by the condition (Y.2) of Sec. 3. Now we fix $\sigma$ as follows. For $a>\{0\}$ we set $\sigma_{a}^{2}=\left(1-\varepsilon^{2|a|}\right)^{-1}$ in accordance with (A.2), with $\varepsilon$ in the range

$$
0<\varepsilon^{2} \leq\left(2+M^{2}\right)^{-1} .
$$

Then we fix $\sigma_{\{0\}}$ (arbitrary large) in accordance with (3.23).

Lemma A.2. Let $a>\{0\}$ and $m_{a}(x):=\max \left(\sigma_{\{0\}}, \sigma_{a}\left|x_{a}\right|\right)=m_{a}\left(x_{a}\right)$. Then

(i) If $x \in C_{a}^{*}(\sigma)$ then $\hat{g}(x, \sigma)=m_{a}\left(x_{a}\right)$.

(ii) $\hat{g}_{a}(x, \sigma)$ has support in $\overline{C_{a}^{*}(\sigma)}$, where $\hat{g}_{a}(x, \sigma)=\sigma_{a}\left|x_{a}\right| \theta\left(\sigma_{a}\left|x_{a}\right|-\sigma_{\{0\}}\right)$.

(iii) If $x \in C_{a}(\sigma)$ then $\hat{g}(x, \sigma)=\max _{b \leq a}\left(m_{b}\left(x_{b}\right)\right)=\hat{g}\left(x_{a}, \sigma\right)$ and $\hat{g}_{\{0\}}(x, \sigma)=\sigma_{\{0\}} \theta\left(\sigma_{\{0\}}-\hat{g}\left(x_{a}, \sigma\right)\right)=\hat{g}_{\{0\}}\left(x_{a}, \sigma\right)$.

(iv) If $x \in C_{a}(\sigma)$ and $b>\{0\}$ then $\hat{g}_{b}(x, \sigma)=0$ unless $b \leq a$. In that case $\hat{g}_{b}(x, \sigma)=m_{b}\left(x_{b}\right) \theta\left[m_{b}\left(x_{b}\right)-\hat{g}\left(x_{a}, \sigma\right)\right]$, in particular $\hat{g}_{b}(x, \sigma)=\hat{g}_{b}\left(x_{a}, \sigma\right)$.

Proof. (i) Let $x \in C_{a}^{*}(\sigma)$ and $\hat{g}(x, \sigma)>\sigma_{\{0\}}$ (else the statement is trivial). It suffices to restrict $x$ to the set where the maximal piece of $\hat{g}$ is unique, i.e.

$$
\hat{g}(x, \sigma)=\sigma_{b}\left|x_{b}\right|>\sigma_{c}\left|x_{c}\right|
$$

for some $b>0$ and all $c \neq b$, which implies $x \in C_{b}(\sigma)$. We have to show that $b=a . \quad b<a$ is excluded since $x \in C_{a}^{*}(\sigma) . \quad b \cap a<a, b$ is equally excluded since then $x \in C_{a \cap b}(\sigma)$. Finally $b>a$ is excluded since (A.4) states that $\sigma_{b}\left|x_{b}\right|>\sigma_{a}\left|x_{a}\right|$ which contradicts $x \in C_{a}(\sigma)$.

(ii) follows from (i) since the sets $C_{a}^{*}(\sigma)$ form a disjoint covering of $X$, and since the decomposition of $\hat{g}$ into maximal pieces is unique. (iii) follows from (i) since

$$
C_{a}(\sigma)=\bigcup_{b \leq a} C_{b}^{*}(\sigma)
$$


and since $x_{b}=\left(x_{a}\right)_{b}$ for $b \leq a$. (iv) follows from (iii) by reading off the maximal pieces of $\hat{g}$ on $C_{a}(\sigma)$.

To perform the regularisation (3.24) explicitly we treat the parameters $\sigma_{a}$ as variables, restricted to disjoint intervals

$$
\sigma_{a}^{-}<\sigma_{a}<\sigma_{a}^{+},
$$

which requires $\sigma_{a}^{+}<\sigma_{b}^{-}$for $b<a$. A possible choice is

$$
\left(\sigma_{a}^{-}\right)^{2}=\left(1-\varepsilon^{2|a|}\right)^{-1} ; \quad\left(\sigma_{a}^{+}\right)^{2}=\left(1-2 \varepsilon^{2|a|}\right)^{-1}
$$

for $a>\{0\}$, with $\varepsilon$ in the range (A.3). $\sigma_{\{0\}}^{ \pm}$is fixed with $\sigma_{\{0\}}^{+}>\sigma_{\{0\}}^{-}>\sigma_{a}^{+} \forall a>\{0\}$. For $a>\{0\}$ we define

$$
C_{a}^{ \pm}=\left\{x\left|\sigma_{a}^{ \pm}\right| x_{a}\left|>\sigma_{b}^{\mp}\right| x_{b} \mid \forall b>a\right\}
$$

so that

$$
C_{a}^{-} \subset C_{a}(\sigma) \subset C_{a}^{+}
$$

for all $\sigma$ allowed by (A.5). As in the proof of Lemma A.1 it follows from (A.3) that

$$
C_{a}^{+} \cap C_{b}^{+} \subset C_{a \cap b}^{-} \quad \text { if } a \cap b<a, b,
$$

so that the functions $\hat{g}(x, \sigma)$ have the properties listed in Lemma A.2 for all allowed $\sigma$.

Now we pick arbitrary weight functions $0 \leq \delta_{a} \in C_{0}^{\infty}\left(\sigma_{a}^{-}, \sigma_{a}^{+}\right)$, normalized to $\int d s \delta_{a}(s)=1$, and we set $j_{a}(s)=\int_{-\infty}^{s} d t \delta_{a}(t)$. The regularisation of $\hat{g}(x, \sigma)$ is then defined by

$$
g(x)=\int \hat{g}(x, \sigma) \prod_{c \in L}\left(\delta_{c}\left(\sigma_{c}\right) d \sigma_{c}\right),
$$

and similarly for $g_{a}(x)$. For $a>\{0\}$ we insert the explicit form

$$
\hat{g}_{a}(x, \sigma)=\sigma_{a}\left|x_{a}\right| \theta\left(\sigma_{a}\left|x_{a}\right|-\sigma_{\{0\}}\right) \prod_{\{0\}>b \neq a} \theta\left(\sigma_{a}\left|x_{a}\right|-\sigma_{b}\left|x_{b}\right|\right),
$$

which gives

$$
g_{a}(x)=\left|x_{a}\right| \int d s s \delta_{a}(s) j_{0}\left(s\left|x_{a}\right|\right) \prod_{\{0\}<b \neq a} j_{b}\left(\frac{s\left|x_{a}\right|}{\left|x_{b}\right|}\right)
$$

and similarly for $g_{\{0\}}(x)$. These formulas only serve to exhibit the smoothness of the functions $g$ and $g_{a}$. All the other relevant properties of $g$ and $g_{a}$ follow directly from Lemma A.2 via (A.14) and (A.16). The result is:

Lemma A.3. The function $g$ constructed above is a Yafaev function with respect to the cones $C_{a}^{-}$(A.6). Moreover, $g$ has a decomposition (3.25) into smooth functions $g_{a}$ with the following properties. There exist constants $0<\bar{\sigma}_{a}$ and $0<R^{-}<R^{+}$ such that 
(i) For $a=\{0\}$ :

$$
\begin{aligned}
& \operatorname{supp}\left(g_{\{0\}}\right) \subset\left\{|x|<R^{+}\right\} ; \\
& g_{\{0\}}(x)=g(0)=\bar{\sigma}_{\{0\}} \text { if }|x|<R^{-} ; \\
& g_{\{0\}}(x)=g_{\{0\}}\left(x_{b}\right) \text { on } C_{b}^{-} \quad \forall b>\{0\} .
\end{aligned}
$$

(ii) For $a>\{0\}$ :

$$
\begin{aligned}
& \operatorname{supp}\left(g_{a}\right) \subset\left\{|x|>R^{-}\right\} \cap\left(C_{a}^{+} \backslash \bigcup_{b<a} C_{b}^{-}\right) ; \\
& g_{a}(x)=g(x)=\bar{\sigma}_{a}\left|x_{a}\right| \text { on } C_{a}^{-} \backslash \bigcup_{b<a} C_{b}^{+} ; \\
& g_{a}(x) \text { is homogeneous of degree } 1 \text { for }|x|>R^{+} ; \\
& g_{a}(x)=g_{a}\left(x_{b}\right) \text { on } C_{b}^{-} \quad \forall b>\{0\} .
\end{aligned}
$$

Corollary A.1. There exists $\lambda>0$ such that:

(i) If $a>\{0\}$ and $x \in \operatorname{supp}\left(g_{a}\right)$, then $|x|_{a} \geq \lambda|x| \geq \lambda R^{-}$.

(ii) If $a \geq\{0\}, b>\{0\}$ and $x \in \operatorname{supp}\left(\nabla g_{a}\right) \cap\left(C_{b}^{-}\right)^{*}$, then

$$
\nabla g_{a}(x) \in b \text { and }|x|_{b} \geq \lambda|x| \geq \lambda R^{-} .
$$

Proof. (i) follows from

$$
C_{b}^{-} \cap\left(C_{a}^{+} \backslash \bigcup_{c<a} C_{c}^{-}\right)=\phi \text { if } b \cap a<a,
$$

which is proved like (3.13) using (A.8). (ii) follows directly from the fact that $g_{a}(x)=g_{a}\left(x_{b}\right)$ on $C_{b}^{-}$.

Lemma A.4. Suppose that $f$ is a function on $X$ which has all the properties of a Yafaev function except convexity: $f$ is smooth; $f(x)=f\left(x_{a}\right)$ near a (in particular $f(x)=0$ for $\left.|x|<R_{1}\right)$; and $f(\lambda x)=\lambda f(x)$ for $|x|>R_{2}(\lambda>1)$. Then there exists a Yafaev function $\tilde{g}$ such that the Hessians of $f$ and $\tilde{g}$ satisfy

$$
\pm f^{\prime \prime}(x) \leq \tilde{g}^{\prime \prime}(x) \quad \forall x .
$$

Proof. For any fixed $y \in X, y \neq 0$ we first construct a local bound, i.e. a Yafaev function $\tilde{g}$ such that (A.10) holds for $x$ near $y$. Since $y \in a^{*}$ for some $a>\{0\}$ there exists a Yafaev function $g$ with $g(x)=\sigma\left|x_{a}\right|$ near $y$ so that

$$
g^{\prime \prime}(x)=\frac{\sigma}{\left|x_{a}\right|} \pi_{a}(x),
$$

where $\pi_{a}(x)$ is the projection of $X$ into $a$ given by

$$
\xi \rightarrow \xi_{a}-\frac{1}{\left|x_{a}\right|^{2}}\left(x_{a} \cdot \xi\right) x_{a}
$$


which has $\operatorname{rank} \operatorname{dim}(a)-1$. Since $f(x)=f\left(x_{a}\right)$ near $y \in a, f^{\prime \prime}(x)$ also maps $X$ into $a$. However, $f^{\prime \prime}(x)$ may have full rank $=\operatorname{dim}(a)$ in the shell $R_{1}<|x|<R_{2}$ where $f$ is not homogeneous. For this reason we first replace $g(x)$ by $\max (g(y), g(x))$ and regularize this to

$$
\tilde{g}(x)=\int d s \delta(s)[s \theta(s-g(x))+g(x) \theta(g(x)-s)],
$$

where $0 \leq \delta \in C_{0}^{\infty}(R)$ is a regularized $\delta$-distribution supported near $g(y)$. By construction $\tilde{g}$ is a Yafaev function with Hessian

$$
\tilde{g}^{\prime \prime}(x)=\sigma^{2}\left(1_{a}-\pi_{a}(x)\right) \delta(g(x))+\frac{\sigma}{\left|x_{a}\right|} \pi_{a} \int_{-\infty}^{g(x)} d s \delta(s),
$$

which is strictly positive near $y$. Multiplying $\tilde{g}$ with a suitable constant we thus have $\pm f^{\prime \prime}(x) \leq \tilde{g}^{\prime \prime}(x)$ in some neighbourhood of $y$. Since the Yafaev functions form a positive cone this estimate extends to all $x$ in the compact shell $R_{1} \leq|x| \leq R_{2}$ by summing over finitely many local bounds. The resulting estimate then extends to $|x|>R_{2}$ by scaling $x \rightarrow \lambda x(\lambda>0)$. In fact (A.11) implies

$$
g^{\prime \prime}(\lambda x) \geq \text { const. } \frac{\pi_{a}(x)}{\lambda\left|x_{a}\right|}, \quad \text { while } \quad \pm f^{\prime \prime}(\lambda x) \leq \text { const. } \frac{\pi_{a}(x)}{\lambda\left|x_{a}\right|}
$$

by homogeneity. For $|x|<R_{1} f^{\prime \prime}(x)=0$ so that (A.10) is trivial.

\section{Appendix B. Commutator Expansions}

Functions of self-adjoint operators. A convenient operator calculus for functions $f(A)$ of self-adjoint operators $A$ can be based on a formula given by Helffer and Sjöstrand [23]:

$$
f(A)=-\frac{1}{2 \pi} \int_{R^{2}}(z-A)^{-1} \partial_{\bar{z}} \tilde{f}(z) d x d y
$$

where $z=x+i y ; \partial_{\bar{z}}=\partial_{x}+i \partial_{y}$. Here $f$ is some given complex function on $R$, and $\tilde{f}$ a largely arbitrary extension of $f$ to the complex plane, which must be almost analytic in the sense that it satisfies the Cauchy-Riemann equations on the real axis:

$$
\partial_{\bar{z}} \tilde{f}(z)=0 \text { for } z \in R
$$

We abbreviate (B.1) by writing:

$$
f(A)=\int d \tilde{f}(z)(z-A)^{-1} ; \quad d \tilde{f}(z) \equiv-\frac{1}{2 \pi} \partial_{\bar{z}} \tilde{f}(z) d x d y .
$$

For example, if $f \in C_{0}^{2}(R)$, we can construct the almost analytic extension

$$
\tilde{f}(z)=\left(f(x)+i y f^{\prime}(x)\right) \chi(z)
$$

in $C_{0}^{1}(C)$ by taking $\chi \in C_{0}^{\infty}(C)$ with $\chi=1$ on some complex neighbourhood of $\operatorname{supp}(f)$. 
Lemma B.1. (B.3) holds for $f \in C_{0}^{2}(R)$ if $\tilde{f} \in C_{0}^{1}(C)$ is an almost analytic extension of $f$, the integral being absolutely convergent in norm sense.

Proof. $\partial_{\bar{z}} \tilde{f}$ has compact support and vanishes on the real axis, so that $\left|\partial_{\bar{z}} \tilde{f}(z)\right| \leq$ const. $|y|$. On the other hand, $\left\|(z-A)^{-1}\right\| \leq|y|^{-1}$. Therefore the integral (B.3) converges absolutely in norm sense, and it suffices to prove that

$$
f_{\epsilon}(t) \equiv \int_{|y|>\epsilon} d \tilde{f}(z)(z-t)^{-1}
$$

converges pointwise to $f(t)$ for $t \in R$ as $\epsilon \searrow 0$. Since $(z-t)^{-1}$ is analytic for $z \notin R$ we have $\partial_{\bar{z}}(z-t)^{-1}=0$. Therefore we obtain after partial integrations in $x$ and $y$ :

$$
f_{\epsilon}(t)=\int d x g_{\epsilon}(t, x) ; \quad g_{\epsilon}(t, x)=\left.\frac{1}{2 \pi i} \tilde{f}(x+i y)(x+i y-t)^{-1}\right|_{y=\epsilon} ^{y=-\epsilon} .
$$

Expanding $\tilde{f}(x \pm i \epsilon)=f(x) \pm i \epsilon f^{\prime}(x)+O\left(\epsilon^{2}\right)$ we find:

$$
g_{\epsilon}(t, x)=\frac{1}{\pi} f(x) \frac{\epsilon}{(x-t)^{2}+\epsilon^{2}}-\frac{1}{2 \pi} f^{\prime}(x) \frac{2 \epsilon(x-t)}{(x-t)^{2}+\epsilon^{2}}+O(\epsilon) .
$$

The second term is bounded by $(1 / 2 \pi)\left|f^{\prime}(x)\right|$ and vanishes pointwise for $x \neq t$ as $\epsilon \rightarrow 0$. Therefore

$$
\lim _{\epsilon \searrow 0} f_{\epsilon}(t)=\lim _{\epsilon \searrow 0} \frac{1}{\pi} \int d x f(x) \frac{\epsilon}{(x-t)^{2}+\epsilon^{2}}=f(t) .
$$

Now let $f \in C^{n+2}(R)$ for some $n \geq 0$. Following [28] (see also [6]) we generalize (B.4) by constructing the almost analytic extension

$$
\tilde{f}(z)=\chi\left(\frac{y}{\langle x\rangle}\right) \sum_{k=0}^{n+1} f^{(k)}(x) \frac{(i y)^{k}}{k !}
$$

where $\langle x\rangle=\left(1+x^{2}\right)^{1 / 2} ; \chi \in C_{0}^{\infty}(R)$ and $\chi=1$ on some open interval $\ni 0$. From

$$
\begin{aligned}
& \partial_{\bar{z}} \sum_{k=0}^{n+1} f^{(k)}(x) \frac{(i y)^{k}}{k !}=f^{(n+2)}(x) \frac{(i y)^{n+1}}{(n+1) !} \\
& \partial_{\bar{z}} \chi\left(\frac{y}{\langle x\rangle}\right)=\frac{1}{\langle x\rangle} \chi^{\prime}\left(\frac{y}{\langle x\rangle}\right)\left(i-\frac{x y}{\langle x\rangle^{2}}\right)
\end{aligned}
$$

we obtain the estimate:

$$
\left|\partial_{\bar{z}} \tilde{f}(z)\right| \leq\left|\chi\left(\frac{y}{\langle x\rangle}\right) \frac{(i y)^{n+1}}{(n+1) !}\right|\left|f^{(n+2)}(x)\right|+\sum_{k=0}^{n+1}\left|\rho\left(\frac{y}{\langle x\rangle}\right) \frac{y^{k}}{k !}\right|\left|\frac{1}{\langle x\rangle} f^{(k)}(x)\right|,
$$

where $\rho(t)=\left|\chi^{\prime}(t)\right|\langle t\rangle$ has compact support $\not \ngtr 0$. Therefore:

$$
\int d y\left|\partial_{\bar{z}} \tilde{f}(z)\right||y|^{-p-1} \leq \text { const. } \sum_{k=0}^{n+2}\langle x\rangle^{k-p-1}\left|f^{(k)}(x)\right|,
$$


since the integrability of the first term in (B.6) against $|y|^{-p-1}$ requires $p \leq n$. Defining the norms

$$
\|f\|_{m}=\int d x\langle x\rangle^{m}|f(x)|
$$

we obtain

$$
\int|d \tilde{f}(z)||\operatorname{Im}(z)|^{-p-1} \leq \text { const. } \sum_{k=0}^{n+2}\left\|f^{(k)}\right\|_{k-p-1}
$$

for $p=0 \cdots n$, provided that

$$
\left\|f^{(k)}\right\|_{k-p-1}<\infty \text { for } k=0 \cdots n+2 .
$$

Lemma B.2. Let $f \in C^{n+2}(R), n \geq 0$, and suppose that (B.9) holds for $p=$ $0 \cdots n$. Let $\tilde{f}(z)$ be given by (B.5). Then

$$
\frac{1}{p !} f^{(p)}(A)=\int d \tilde{f}(z)(z-A)^{-p-1}
$$

for $p=0 \cdots n$ and for all selfadjoint operators $A$, where by (B.8) the integral converges absolutely in norm sense and is bounded uniformly in $A$.

Proof. To prove (B.10) we first assume $f \in C_{0}^{n+2}(R)$. Then $\partial_{x}^{p} \tilde{f}(z)$ is an almost analytic extension of $f^{(p)}(x)$ in the sense of Lemma B.1, so that

$$
f^{(p)}(A)=\int d\left(\partial_{x}^{p} \tilde{f}(z)\right)(z-A)^{-1}=p ! \int d \tilde{f}(z)(z-A)^{-p-1}
$$

by partial integration in $x$. Now let $f \in C^{n+2}(R)$ obey (B.9). Then (B.10) holds for $f$ replaced by $f_{m}(x)=f(x) \chi(x / m), \chi \in C_{0}^{\infty}(R)$ with $\chi(x)=1$ near $x=0$. It is easy to see that

$$
\lim _{m \rightarrow \infty}\left\|f^{(k)}-f_{m}^{(k)}\right\|_{k-p-1}=0 ; \quad k=0 \cdots n+2 .
$$

Moreover, $f_{m}^{(p)}(x)$ is uniformly bounded in terms of $\left\|f_{m}^{(p+1)}\right\|_{0}$ and converges pointwise to $f^{(p)}(x)$ as $m \rightarrow \infty$. Therefore (B.10) is preserved in this limit.

Commutator Expansions. Now we derive expansion formulae for commutators with remainder whose prototype was introduced in [45] (see also [2] for a different version). We consider two bounded operators $H$ and $A=A^{*}$. Multiple commutators are defined recursively by

$$
a d_{A}^{(k)}(H)=\left[a d_{A}^{(k-1)}(H), A\right] ; \quad a d_{A}^{(0)}(H)=H .
$$

Then

$$
\left[H,(z-A)^{-1}\right]=(z-A)^{-1}[H, A](z-A)^{-1},
$$

and more generally:

$$
\left[a d_{A}^{(k-1)}(H),(z-A)^{-1}\right]=(z-A)^{-1} a d_{A}^{(k)}(H)(z-A)^{-1} .
$$


Starting from (B.11) we use (B.12) to commute the rightmost resolvent $(z-A)^{-1}$ systematically to the left, obtaining:

$$
\left[H,(z-A)^{-1}\right]=\sum_{k=1}^{n-1}(z-A)^{-k-1} a d_{A}^{(k)}+(z-A)^{-n} a d_{A}^{(n)}(H)(z-A)^{-1} .
$$

Let $f \in C_{0}^{\infty}(R)$, and let $\tilde{f}$ be the almost analytic extension (B.5). From (B.13) and Lemma B.2 we find the commutator expansion:

$$
\begin{aligned}
{[H, f(A)] } & =\sum_{k=1}^{n-1} \frac{1}{k !} f^{(k)}(A) a d_{A}^{(k)}(H)+R_{n} \\
R_{n} & =\int d \tilde{f}(z)(z-A)^{-n} a d_{A}^{(n)}(H)(z-A)^{-1}
\end{aligned}
$$

with the estimate

$$
\left\|R_{n}\right\| \leq \text { const. }\left\|a d_{A}^{(n)}(H)\right\| \sum_{k=0}^{n+2}\left\|f^{(k)}\right\|_{k-n} .
$$

Similarly, we could have commuted the resolvents $(z-A)^{-1}$ systematically to the right, arriving at

$$
\begin{aligned}
{[H, f(A)] } & =\sum_{k=1}^{n-1} \frac{1}{k !}(-1)^{k-1} a d_{A}^{(k)}(H) f^{(k)}(A)+R_{n} \\
R_{n} & =(-1)^{n-1} \int d \tilde{f}(z)(z-A)^{-1} a d_{A}^{(n)}(H)(z-A)^{-n}
\end{aligned}
$$

and with the same estimate (B.15). Combining the two expansions we also find a useful symmetric form for $n=2$ :

$$
\begin{aligned}
{[H, f(A)] } & =\frac{1}{2}\left[f^{\prime}(A) a d_{A}^{(1)}(H)+a d_{A}^{(1)} f^{\prime}(A)\right]+R_{2} \\
R_{2} & =\frac{1}{2} \int d \tilde{f}(z)(z-A)^{-1}\left[(z-A)^{-1}, a d_{A}^{(2)}(H)\right](z-A)^{-1} \\
& =-\frac{1}{2} \int d \tilde{f}(z)(z-A)^{-2} a d_{A}^{(3)}(H)(z-A)^{-2}
\end{aligned}
$$

As in the proof of Lemma B.2, these expansions extend to all bounded $C^{\infty}$-functions $f$ with bounded derivatives, as long as the norms arising in (B.15) remain finite.

Commutator Estimates. We will often deal with commutators of the form $[g(H), f(A)]$ for unbounded selfadjoint operators $H, A$. We assume that $g \in C_{0}^{\infty}(R)$. Then the representation

$$
[g(H), A]=\int d \tilde{g}(z)(z-H)^{-1}[H, A](z-H)^{-1}
$$


requires an almost analytic extension $\tilde{g}(z)$ of the form (B.5) with $n \geq 1$. The integral is well defined if $[H, A]$ is $H$-bounded, since then

$$
\left\|[H, A](z-H)^{-1}\right\| \leq \text { const. }(1+|z|)|\operatorname{Im}(z)|^{-1},
$$

where the factor $|z|$ is harmless since $\tilde{g}$ has compact support. Similarly, we can deal with

$$
a d_{A}^{(k)}(g(H))=\int d \tilde{g}(z) a d_{A}^{(k)}\left((z-H)^{-1}\right),
$$

writing out

$$
\begin{aligned}
a d_{A}^{(1)}\left((z-H)^{-1}\right)= & (z-H)^{-1} a d_{A}^{(1)}(H)(z-H)^{-1} ; \\
a d_{A}^{(2)}\left((z-H)^{-1}\right)= & 2(z-H)^{-1} a d_{A}^{(1)}(H)(z-H)^{-1} a d_{A}^{(1)}(H)(z-H)^{-1} \\
& +(z-H)^{-1} a d_{A}^{(2)}(H)(z-H)^{-1} ;
\end{aligned}
$$

and so forth. Therefore, if $a d_{A}^{(k)}(H)$ is $H$-bounded for $1 \leq k \leq n$, we have

$$
\left\|a d_{A}^{(n)}\left((z-H)^{-1}\right)\right\| \leq \text { const. } \sum_{p=1}^{n}|\operatorname{Im}(z)|^{-p-1}
$$

on $\operatorname{supp}(\tilde{g})$. To use (B.20) we take an almost analytic extension $\tilde{g}(z)$ of the form (B.5) for the given $n$, which leads to a bound:

$$
\left\|a d_{A}^{(n)}(g(H))\right\| \leq \text { const. } \sum_{p=1}^{n} \sum_{k=0}^{n+2}\left\|g^{(k)}\right\|_{k-p-1} .
$$

Now we can discuss

$$
[g(H), f(A)]=\int d \tilde{f}(z)(z-A)^{-1}[g(H), A](z-A)^{-1} .
$$

If $[H, A]$ is $H$-bounded, then $[g(H), A]$ is bounded. $\tilde{f}(z)$ can be taken of the form (B.5) with $n=1$, and the convergence of the integral (B.22) requires that

$$
\left\|f^{(k)}\right\|_{k-2}<\infty \text { for } k=0 \cdots 3 .
$$

This already allows $f(x)$ to grow like $|x|^{p}$ with $p<1$, if also $f^{(k)}(x)=O\left(|x|^{p-k}\right)$ for $k \leq 3$. Suppose now that $a d_{A}^{(k)}(H)$ is $H$-bounded for $k \leq n$. Then $a d_{A}^{(k)}(g(H))$ is bounded for $k \leq n$ and we can represent the commutator $[g(H), f(A)]$ by an expansion, like

$$
\begin{aligned}
{[g(H), f(A)] } & =\sum_{k=1}^{n-1} \frac{1}{k !} f^{(k)}(A) a d_{A}^{(k)}(g(H))+R_{n} \\
R_{n} & =\int d \tilde{f}(z)(z-A)^{-n} a d_{A}^{(n)}(g(H))(z-A)^{-1} .
\end{aligned}
$$


Here $\tilde{f}(z)$ must be of the form (B.5) for the given $n$, but the convergence of the integral only requires that

$$
\left\|f^{(k)}\right\|_{k-n-1}<\infty \quad \text { for } k=0 \cdots n+1 .
$$

This allows $f(x)$ to grow like $|x|^{p}, p<n$, with corresponding slower growth of the derivatives.

\section{Appendix C. Estimates for the Long-Range Case}

Here we derive additional propagation estimates for the dynamics $U_{t}: \psi \rightarrow \psi_{t}$ generated by $H_{t}=H+W_{t}(x)$. These estimates are used in Sec. 8 and (indirectly) in Sec. 9. They are based on the hypothesis

$$
\begin{aligned}
& \nabla I_{a}(x)=O\left(|x|_{a}^{-\mu-1}\right) ; \\
& \nabla W_{t}(x) \leq \text { const. }(1+|x|)^{-\mu-1} \quad \forall t \quad(\mu>0) .
\end{aligned}
$$

Roughly speaking we show that the region of ballistic motion can be decoupled from that of a subballistic one (Theorem C. 2 and its consequence). This is used in Sec. 8 in order to conclude that the subballistic part of any orbit is a bound state of $H$.

We pick the scaling parameters $\delta$ and $\delta^{\prime}$ in the ranges

$$
\frac{2}{3}<\delta<1 ; \quad \delta(\mu+2)>2, \quad \delta<\delta^{\prime}<1 .
$$

We also define

$$
\begin{aligned}
\Gamma_{t} & :=\gamma_{t}-\partial_{t} g_{t}=\frac{1}{2}\left(\nabla g_{t} \cdot p+p \cdot \nabla g_{t}\right) ; \\
K & :=\frac{1}{2} p^{2}+1 ; \quad\|\psi\|_{H}:=\|\psi\|+\|H \psi\|,
\end{aligned}
$$

and we will use (1.16) to estimate $\left\|K \psi_{t}\right\| \leq$ const. $\|\psi\|_{H}$. With $\chi(x \leq \theta)$ we denote any smoothed characteristic function of the type shown in Fig. 6; in particular $\operatorname{supp}(\chi) \subset(-\infty, \theta]$ and $0 \geq \chi^{\prime} \in C_{0}^{\infty}(R)$.

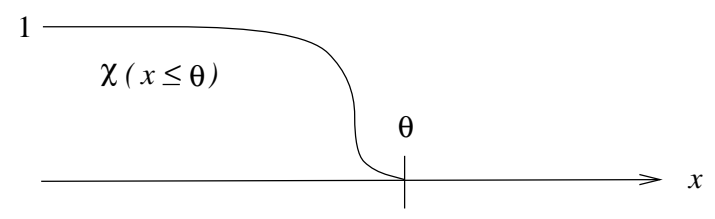

Fig. 6.

If $A$ is a selfadjoint operator then $\chi(A \leq \theta)$ is the corresponding "function" of $A$, and $\chi(A \geq \theta)$ is defined analogously. To estimate commutators involving such operators we will use the methods of Appendix B. The results of Appendix $\mathrm{C}$ are:

Theorem C.1. Let $0<\theta_{1} \leq \theta$. Then, for large $t$ and all $\psi \in D(H)$

$$
\begin{aligned}
& \left\|\chi\left(t^{1-\delta^{\prime}} \Gamma_{t} \leq \theta_{1}\right) \chi\left(t^{-\delta^{\prime}} g_{t} \geq \theta\right) \psi_{t}\right\|^{2} \\
& \quad \leq\left\|\chi\left(t^{-\delta^{\prime}} g_{t} \geq \theta\right) \psi\right\|^{2}+\text { const. } t^{-\rho}\|\psi\|_{H}^{2} \quad(\rho>0) .
\end{aligned}
$$


Remark. $\delta^{\prime}>\delta$ implies that $\chi\left(t^{-\delta^{\prime}} g_{t} \geq \theta\right) \rightarrow 0$ strongly as $t \rightarrow \infty$. Therefore it follows from (C.4) that

$$
\lim _{t \rightarrow \infty} \chi\left(t^{1-\delta^{\prime}} \Gamma_{t} \leq \theta_{1}\right) \chi\left(t^{-\delta^{\prime}} g_{t} \geq \theta\right) \psi_{t}=0 .
$$

This is equivalent to

$$
\begin{gathered}
\lim _{t \rightarrow \infty} \chi\left(t^{-\delta^{\prime}} g_{t} \geq \theta\right) \chi\left(t^{1-\delta^{\prime}} \Gamma_{t} \leq \theta_{1}\right) \psi_{t}=0 \\
\text { since }\left\|\left[t^{1-\delta^{\prime}} \Gamma_{t}, t^{-\delta^{\prime}} g_{t}\right]\right\| \leq \text { const. } t^{1-2 \delta^{\prime}} \rightarrow 0 .
\end{gathered}
$$

Theorem C.2. Let $\theta>0$. Then for large $t$ and with $\rho>1$

$$
D_{t} \chi\left(t^{-\delta^{\prime}} g_{t} \geq \theta\right)=\sum_{k=1}^{6} \pm B_{k}(t)+O\left(t^{-\rho}\right)
$$

in form sense on $D(H)$, with an appropriate sign \pm for each $k$. The quadratic forms $B_{k}(t)$ are positive and satisfy

$$
\int_{1}^{\infty} d t\left\langle B_{k}(t)\right\rangle_{t} \leq \text { const. }\|\psi\|_{H}^{2} \quad \forall \psi \in D(H) .
$$

The symbol $O\left(t^{-\rho}\right)$ denotes a form on $D(H)$ with

$$
\left|\left\langle O\left(t^{-\rho}\right)\right\rangle_{\psi}\right| \leq \text { const. } t^{-\rho}\|\psi\|_{H}^{2} \quad(\rho>1)
$$

for large $t$ and all $\psi \in D(H)$.

Proofs. To prepare the proof of Theorem C.1 we introduce a variable $s \in R^{+}$and we consider the form

$$
\Phi_{t}(s)=f \chi f ; \quad f=f\left(s^{-\delta^{\prime}}\left(g_{s}-c t\right)\right) ; \quad \chi=\chi\left(b \Gamma_{s}\right),
$$

on $D(H)$, with real parameters $b>0$ and $c$ to be adjusted later as functions of $s$. $f$ and $\chi$ are smooth characteristic functions depicted in Fig. 7:

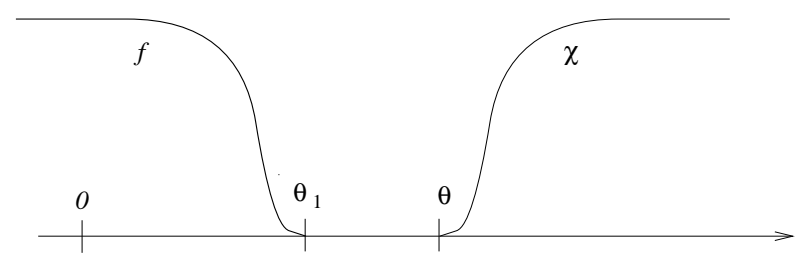

Fig. 7.

Our strategy is to estimate the form

$$
D_{t} \Phi_{t}(s)=f\left(D_{t} \chi\right) f+\left(\left(D_{t} f\right) \chi f+\text { adjoint }\right),
$$


for $0 \leq t \leq s$ ( $s$ large), and then to use this estimate to derive the desired bound for

$$
\left\langle\Phi_{s}(s)\right\rangle_{s}=\left\langle\Phi_{0}(s)\right\rangle_{0}+\int_{0}^{s} d t\left\langle D_{t} \Phi_{t}(s)\right\rangle_{t}
$$

Lemma C.1. For s sufficiently large and for all $\psi \in D(H)$

$$
\left\langle D_{t} \chi\right\rangle_{\psi} \leq \text { const. }\left(b^{3} s^{-3 \delta}+b s^{-\delta(\mu+1)}\right)\|\psi\|_{H}^{2}
$$

with the constant independent of $t, c, b$ and $s$.

Proof. Let $\chi$ be such that $\left|\chi^{\prime}\right|^{1 / 2} \in C_{0}^{\infty}$. We write

$$
D_{t} \chi=i\left[H_{t}, \chi\right]=A+B ; \quad A=i[K, \chi] ; \quad B=i\left[V+W_{t}, \chi\right]
$$

Estimate of $A$ : the symmetric expansion (B.18) for $K^{-1} A K^{-1}=-i\left[K^{-1}, \chi\right]$ reads

$$
\begin{aligned}
K^{-1} A K^{-1}= & -\frac{b}{2}\left(\chi^{\prime} i\left[K^{-1}, \Gamma_{s}\right]+\text { adjoint }\right) \\
& +\frac{i b^{3}}{2} \int d \tilde{\chi}(z)\left(z-b \Gamma_{s}\right)^{-2} a d_{\Gamma_{s}}^{(3)}\left(K^{-1}\right)\left(z-b \Gamma_{s}\right)^{-2} .
\end{aligned}
$$

To estimate these terms we note that

$$
a d_{\Gamma_{s}}^{(k)}(K)=p_{m} G_{m n}^{(k)}(x) p_{n}+G^{(k)}(x),
$$

where $G_{m n}^{(k)}(x)$ and $G^{(k)}(x)$ are polynomials of derivatives of $g_{s}(x)$, supported in $\left\{|x| \geq s^{\delta}\right\}$ and homogeneous in $x$ of degree $-k$ and $-(k+2)$, respectively, for large $|x|$. This leads to

$$
\begin{aligned}
\left\|K^{-1} a d_{\Gamma_{s}}^{(k)}(K) K^{-1}\right\| & \leq \text { const. } s^{-k \delta} \quad \text { and } \\
\left\|a d_{\Gamma_{s}}^{(k)}\left(K^{-1}\right)\right\| & \leq \text { const. } s^{-k \delta} .
\end{aligned}
$$

Therefore the second term in (C.15) is of order $b^{3} s^{-3 \delta}$ in norm as $s \rightarrow \infty$. The first term is symmetrized using $\chi^{\prime} \leq 0$ and

$$
\begin{aligned}
-\frac{1}{2} \chi^{\prime} i\left[K^{-1}, b \Gamma_{s}\right]+\text { adjoint }= & \left|\chi^{\prime}\right|^{1 / 2} i\left[K^{-1}, b \Gamma_{s}\right]\left|\chi^{\prime}\right|^{1 / 2} \\
& +\frac{1}{2}\left[\left|\chi^{\prime}\right|^{1 / 2},\left[\left|\chi^{\prime}\right|^{1 / 2}, i\left[K^{-1}, b \Gamma_{s}\right]\right]\right] .
\end{aligned}
$$

By (C.16) the multiple commutator is of order $b^{3} s^{-3 \delta}$ in norm. Finally, using

$$
i\left[K^{-1}, \Gamma_{s}\right]=-K^{-1} i\left[K, \Gamma_{s}\right] K^{-1}=-K^{-1}\left(p g_{s}^{\prime \prime} p-\frac{1}{4} \Delta^{2} g_{s}\right) K^{-1}
$$

and $\Delta^{2} g_{s}=O\left(s^{-3 \delta}\right)$ we arrive at

$$
K^{-1} A K^{-1}=-Q+O\left(b s^{-3 \delta}+b^{3} s^{-3 \delta}\right)
$$


in norm as $s \rightarrow \infty$, where

$$
Q=b\left|\chi^{\prime}\right|^{1 / 2} K^{-1}\left(p g^{\prime \prime} p\right) K^{-1}\left|\chi^{\prime}\right|^{1 / 2} \geq 0 .
$$

Therefore

$$
\langle A\rangle_{\psi}=-\langle P\rangle_{\psi}+O\left(b s^{-3 \delta}+b^{3} s^{-3 \delta}\right)\|\psi\|_{H}^{2}
$$

on $D(H)$, where $P=K Q K$ is a positive quadratic form.

Estimate of B: By (C.1) the commutator in the representation

$$
B=i b \int d \tilde{\chi}(z)\left(z-b \Gamma_{s}\right)^{-1}\left[V+W_{t}, \Gamma_{s}\right]\left(z-b \Gamma_{s}\right)^{-1}
$$

is of order $s^{-\delta(\mu+1)}$ in norm. Therefore

$$
\|B\| \leq \text { const. } b s^{-\delta(\mu+1)}
$$

uniformly in $t$ if $s$ is sufficiently large. (C.13) now follows from (C.17) and (C.18).

Lemma C.2. If $b c \geq \theta_{1}$ then

$$
\left\langle\left(D_{t} f\right) \chi f+\text { adjoint }\right\rangle_{\psi} \leq \text { const. } b s^{-2 \delta^{\prime}}\|\psi\|^{2}
$$

for all $\psi \in D(H)$, uniformly in $t$.

Proof. Since $f=f\left(s^{-\delta^{\prime}}\left(g_{s}-c t\right)\right)$ is a function of $x$ we can easily compute $D_{t}(f)$ with the result

$$
D_{t} f=s^{-\delta^{\prime}}\left(f^{\prime}\right)^{1 / 2} \cdot\left(\Gamma_{s}-c\right) \cdot\left(f^{\prime}\right)^{1 / 2},
$$

where we have used that $f^{\prime} \geq 0$ and that the commutator of $\Gamma_{s}$ with a function of $x$ is again a function of $x$. In particular, $i\left[\Gamma_{s}, g_{s}\right]=\left(\nabla g_{s}\right)^{2}$ is bounded uniformly in $s$ so that

$$
\left\|\left[\left(f^{\prime}\right)^{1 / 2}, \chi\left(b \Gamma_{s}\right)\right]\right\| \leq \text { const. } b s^{-\delta^{\prime}} .
$$

Using this we arrive at

$$
\begin{aligned}
\left(D_{t} f\right) \chi & =s^{-\delta^{\prime}}\left(f^{\prime}\right)^{1 / 2} \chi\left(b \Gamma_{s}\right) \cdot\left(\Gamma_{s}-c\right) \cdot\left(f^{\prime}\right)^{1 / 2}+O\left(b s^{-2 \delta^{\prime}}\right) ; \\
\left(D_{t} f\right) \chi f+\text { adjoint } & =s^{-\delta^{\prime}} f_{1} \chi\left(b \Gamma_{s}\right) \cdot\left(\Gamma_{s}-c\right) \cdot f_{1}+O\left(b s^{-2 \delta^{\prime}}\right),
\end{aligned}
$$

where $f_{1}^{2}:=\left(f^{2}\right)^{\prime}$. (C.19) now follows by observing that $\chi\left(b \Gamma_{s}\right) \cdot\left(\Gamma_{s}-c\right) \leq 0$ if $b c \geq \theta_{1}$.

Proof of Theorem C.1. By (C.13) and (C.19)

$$
\left\langle D_{t} \Phi_{t}(s)\right\rangle_{t} \leq \text { const. }\left(b^{3} s^{-3 \delta}+b s^{-\delta(\mu+1)}\right)\|\psi\|_{H}^{2}
$$

uniformly in $t$. From (C.12) we thus obtain

$$
\left\langle\Phi_{s}(s)\right\rangle_{s} \leq\left\langle\Phi_{0}(s)\right\rangle_{0}+\text { const. }\left(b^{3} s^{1-3 \delta}+b s^{1-\delta(\mu+1)}\right)\|\psi\|_{H}^{2} .
$$


Now we fix $b=s^{1-\delta^{\prime}} ; c=\theta_{1} s^{\delta^{\prime}-1}$. Then (C.22) reads

$$
\left\langle\Phi_{s}(s)\right\rangle_{s} \leq\left\langle\Phi_{0}(s)\right\rangle_{0}+\text { const. } s^{-\rho}\|\psi\|_{H}^{2},
$$

where $\rho=\min (6 \delta-4, \delta(\mu+2)-2)>0$. Replacing $s$ by $t$ and $\chi$ by $\chi^{2}$ we arrive at (C.4).

For the proof of Theorem C.2 we consider $f=f\left(t^{-\delta^{\prime}} g_{t}\right)$ with the function $f$ shown in Fig. 8 (we identify $f^{2}$ with the function $\chi\left(t^{-\delta^{\prime}} g_{t} \geq \theta\right.$ ) of (C.7)).

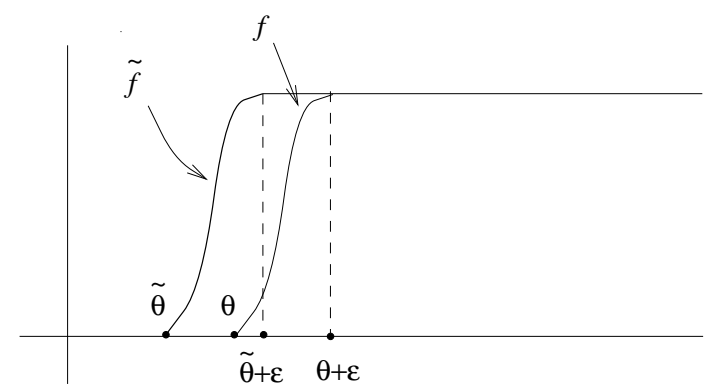

Fig. 8.

Our task is to decompose $D_{t}\left(f^{2}\right)$ into a sum of positive and negative quadratic forms in the sense of (C.8). Like (C.20) we find

$$
D_{t}\left(f^{2}\right)=t^{-1} f_{1} \cdot\left(t^{1-\delta^{\prime}} \Gamma_{t}-\delta^{\prime} t^{-\delta^{\prime}} g_{t}+t^{1-\delta^{\prime}} \partial_{t} g_{t}\right) \cdot f_{1}
$$

with $f_{1}^{2}=\left(f^{2}\right)^{\prime}$. The first step of the decomposition is achieved by inserting a partition of unity

$$
1=\sum_{k=1}^{3} \chi_{k}\left(t^{1-\delta^{\prime}} \Gamma_{t}\right)
$$

with functions $\chi_{k}$ as shown in Fig. 9 .

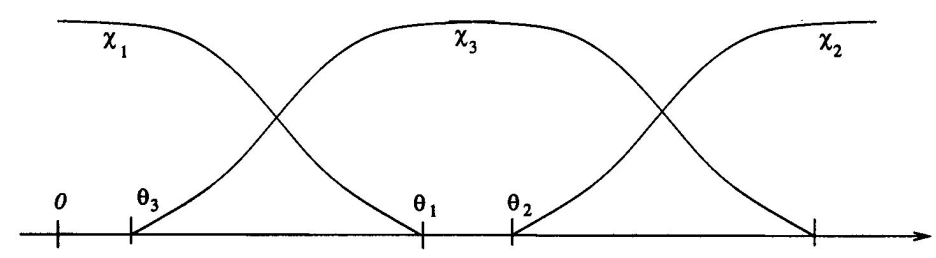

Fig. 9.

The parameters $\theta_{1} \cdots \theta_{3}$ will be selected in the course of the proof. (C.6) allows us to write

$$
D_{t}\left(f^{2}\right)=\sum_{k=1}^{3} t^{-1} f_{1} \chi_{k}^{1 / 2} \cdot\left(t^{1-\delta^{\prime}} \Gamma_{t}-\delta^{\prime} t^{-\delta^{\prime}} g_{t}\right) \cdot \chi_{k}^{1 / 2} f_{1}+O\left(t^{-\rho}\right)
$$


with $O\left(t^{-\rho}\right)$ defined in (C.9). For the same reason the factors $\chi_{k}^{1 / 2}$ and $f_{1}$ can be freely commuted modulo contributions to $O\left(t^{-\rho}\right)$, a fact we shall use without comment in the analysis below. The task of proving Theorem C.2 is now reduced to prove (C.7) separately for each term in the sum (C.24).

Let $\chi$ be one of the functions $\chi_{1}, \chi_{2}$ appearing in (C.24) and let $A_{t}=t^{1-\delta^{\prime}} \Gamma_{t}$. In what follows we have to compute $\partial_{t} \chi\left(A_{t}\right)$. We would like to do that using the functional calculus of Appendix B via

$$
\partial_{t}\left(z-A_{t}\right)^{-1}=-\left(z-A_{t}\right)^{-1}\left(\partial_{t} A_{t}\right)\left(z-A_{t}\right)^{-1} .
$$

In general this expression is ill-defined as an operator (or form) on $D(|p|)$ since we cannot expect that $\left(z-A_{t}\right)^{-1}$ maps $D(|p|)$ into itself. To clarify this point we observe that the commutators

$$
\left[p_{k}, A_{t}\right]=t^{1-\delta-\delta^{\prime}}\left(C_{k \ell} p_{\ell}+C_{k}\right)
$$

are first-order differential operators with bounded coefficients $C_{k \ell}(x, t), C_{k}(x, t)$. Therefore the operators $B_{k}(z)=p_{k}\left(z-A_{t}\right)^{-1}(1+|p|)^{-1}$ obey the coupled equations

$$
\begin{aligned}
B_{k}(z)= & \left(z-A_{t}\right)^{-1} p_{k}(1+|p|)^{-1} \\
& +\left(z-A_{t}\right)^{-1} t^{1-\delta-\delta^{\prime}} C_{k}\left(z-A_{t}\right)^{-1}(1+|p|)^{-1} \\
& +\left(z-A_{t}\right)^{-1} t^{1-\delta-\delta^{\prime}} C_{k \ell} B_{\ell}(z) .
\end{aligned}
$$

This system can be solved by iteration for large $t$ in the region

$$
|\operatorname{Im} z| \geq t^{-\varepsilon} ; \varepsilon<\delta+\delta^{\prime}-1
$$

with a resulting estimate

$$
\left\|p_{k}\left(z-A_{t}\right)^{-1}(1+|p|)^{-1}\right\| \leq \text { const. }|\operatorname{Im} z|^{-1} .
$$

Guided by this result we approximate the function $\chi(s)$ by

$$
\begin{aligned}
& \chi_{t}(s)=\chi(s)-\bar{\chi}_{t}(s) ; \\
& \bar{\chi}_{t}(s)=\int_{|\operatorname{Im} z| \leq t^{-\varepsilon}} d \tilde{\chi}(z)(z-s)^{-1},
\end{aligned}
$$

where $\tilde{\chi}(z)$ is the almost analytic extension of $\chi(x)$ defined by (B.5) with $n$ arbitrary large. Since $\chi^{\prime}(s)$ has compact support it follows from (B.5) and (B.6) that

$$
\bar{\chi}_{t}(s)=\text { const. } \int_{|y| \leq t^{-\varepsilon}} d x d y \chi^{(n+2)}(x) y^{n+1}(x+i y-s)^{-1}
$$

for $t$ sufficiently large. In particular, $\bar{\chi}_{t}(s)$ (which depends on $n$ ) has arbitrary fast time-decay

$$
\sup _{s}\left|\bar{\chi}_{t}(s)\right| \leq \text { const. } t^{-n \varepsilon}
$$

for large $t$. 
The benefit of the approximation (C.27) is that - in contrast to $\chi\left(A_{t}\right)$ - the operator $\chi_{t}\left(A_{t}\right)$ has a time derivative for large $t$ given by

$$
\partial_{t} \chi_{t}\left(A_{t}\right)=-\int_{|\operatorname{Im} z| \geq t^{-\varepsilon}} d \tilde{\chi}(z)\left(z-A_{t}\right)^{-1}\left(\partial_{t} A_{t}\right)\left(z-A_{t}\right)^{-1}+O\left(t^{-n \varepsilon}\right)
$$

on $D(|p|)$, where

$$
\partial_{t} A_{t}=\left(1-\delta^{\prime}\right) t^{-1} A_{t}+t^{1-\delta^{\prime}} \partial_{t} \Gamma_{t} .
$$

To prove this we first remark that the integral in (C.30) is convergent due to (B.6) and the estimate (C.26). Next we note that the formula

$$
\frac{1}{h}\left[\chi_{t}\left(A_{t+h}\right)-\chi_{t}\left(A_{t}\right)\right]=-\int_{|\operatorname{Im} z| \geq t^{-\varepsilon}} d \tilde{\chi}(z)\left(z-A_{t+h}\right)^{-1} \frac{1}{h}\left(A_{t+h}-A_{t}\right)\left(z-A_{t}\right)^{-1}
$$

holds on $D(|p|)$ if $\chi \in C_{0}^{\infty}(R)$ and extends (as a strong limit on $D(|p|)$ ) by continuity in $\chi$ to our case where only $\chi^{\prime} \in C_{0}^{\infty}(R)$. For $h \rightarrow 0$ this shows the existence of the first term in

$$
\partial_{t}\left(\chi_{t}\left(A_{t}\right)\right)=\partial_{s}\left(\chi_{t}\left(A_{s}\right)\right)_{s=t}-\left(\partial_{t} \bar{\chi}_{t}\right)\left(A_{t}\right),
$$

while the second term is of order $t^{-n \varepsilon-\varepsilon-1}$ by (C.28). This proves (C.30). As our principal tool we first estimate the form

$$
D_{t}\left(f \chi_{t} f\right)=f\left(D_{t} \chi_{t}\right) f+\left(\left(D_{t} f\right) \chi_{t} f+\text { adjoint }\right),
$$

where $\chi$ is one of the functions $\chi_{1}, \chi_{2}$ in (C.24), $\chi_{t}=\chi_{t}\left(A_{t}\right)$ and $f=f\left(t^{-\delta^{\prime}} g_{t}\right)$ as before. Since $\chi_{1}^{\prime}$ and $\chi_{2}^{\prime}$ have opposite signs we distinguish two cases:

Lemma C.3. Let $\pm \chi^{\prime} \geq 0$. Then, correspondingly,

$$
f\left(D_{t} \chi_{t}\right) f= \pm P \pm\left(1-\delta^{\prime}\right) t^{-1} f\left|\chi_{t}^{\prime}\right| t^{1-\delta^{\prime}} \Gamma_{t} f+O\left(t^{-\rho}\right), \quad \rho>1,
$$

on $D(H)$, where $P$ is a positive quadratic form on $D(H)$, and where $O\left(t^{-\rho}\right)$ is defined by (C.9).

Proof. The first part of the proof is almost the same as the proof of Lemma C.1. Compared with (C.14) we now have

$$
D_{t} \chi_{t}=A+B+C
$$

with

$$
A=i\left[K, \chi_{t}\right] ; \quad B=i\left[V+W_{t}, \chi_{t}\right]
$$

and with the additional term $C=\partial_{t} \chi_{t}$. Due to (C.29) the contributions of $A$ and $B$ can be estimated as before with $\chi_{t}$ in place of $\chi$, replacing $s$ by $t, b$ by $t^{1-\delta^{\prime}}$ and taking the sign of $\chi^{\prime}$ into account. Corresponding to (C.13) the resulting error is 
of order $t^{-\rho}$ with $\rho=\min (6 \delta-3, \delta(\mu+2)-1)>1$. For the extra contribution of $C=\partial_{t} \chi_{t}$ we obtain from (C.30):

$$
\begin{aligned}
& f\left(\partial_{t} \chi_{t}\right) f=D+E, \quad \text { where } \\
D= & -\left(1-\delta^{\prime}\right) t^{-1} f A_{t} \int_{|\operatorname{Im} z| \geq t^{-\varepsilon}} d \tilde{\chi}(z)\left(z-A_{t}\right)^{-2} f+O\left(t^{-n \varepsilon}\right) \\
= & \left(1-\delta^{\prime}\right) t^{-1} f A_{t} \chi^{\prime}\left(A_{t}\right) f+O\left(t^{-n \varepsilon}\right) ; \\
E= & t^{1-\delta^{\prime}} \int_{|\operatorname{Im} z| \geq t^{-\varepsilon}} d \tilde{\chi}(z)\left(z-A_{t}\right)^{-1}\left[A_{t}, f\right]\left(z-A_{t}\right)^{-1}\left(\partial_{t} \Gamma_{t}\right) \\
& \cdot\left(z-A_{t}\right)^{-1}\left[f, A_{t}\right]\left(z-A_{t}\right)^{-1} .
\end{aligned}
$$

In the last expression we used that $f \cdot\left(\partial_{t} \Gamma_{t}\right)=0$ (i.e. $\partial_{t} g_{t}=0$ on supp $f$ ) for large $t$. As a quadratic form on $D(H), E$ is of order $t^{-\rho}$ in the sense of (C.7) with $\rho=5 \delta^{\prime}-2>1$. This follows from the estimate (C.26) since $\partial_{t} \Gamma_{t}$ is of order $t^{-1}$ relative to $|p|$ and since $\left\|\left[A_{t}, f\right]\right\|$ is of order $t^{1-2 \delta^{\prime}}$. Consequently we have

$$
f\left(\partial_{t} \chi_{t}\right) f=\left(1-\delta^{\prime}\right) t^{-1} f A_{t} \chi^{\prime}\left(A_{t}\right) f+O\left(t^{-\rho}\right)
$$

with $\rho>1$, which together with the remark above concerning the terms $A$ and $B$ implies (C.31).

Lemma C.4. Let $f_{1}^{2}=\left(f^{2}\right)^{\prime}$. Then

$$
\left(D_{t} f\right) \chi_{t} f+\text { adjoint }=t^{-1} f_{1} \chi^{1 / 2} \cdot\left(t^{1-\delta^{\prime}} \Gamma_{t}-\delta^{\prime} t^{-\delta^{\prime}} g_{t}\right) \cdot \chi^{1 / 2} f_{1}+O\left(t^{-\rho}\right) .
$$

Proof. Here $\chi_{t}$ can be replaced by $\chi$ within the error $O\left(t^{-\rho}\right), \rho>1$. Then the estimate (C.36) corresponds to (C.21). The only difference in the proof is that now

$$
\partial_{t} f=-\delta^{\prime} t^{-1-\delta^{\prime}} f^{\prime} g_{t}+t^{-\delta^{\prime}} f^{\prime} \partial_{t} g_{t}
$$

where the last term vanishes exactly for sufficiently large $t$.

Proof of Theorem C.2. By the results so far we have for $\pm \chi^{\prime} \geq 0$ :

$$
\begin{aligned}
D_{t}\left(f \chi_{t} f\right)= & \pm P \\
& \pm\left(1-\delta^{\prime}\right) t^{-1} f\left|\chi^{\prime}\right| \cdot\left(t^{1-\delta^{\prime}} \Gamma_{t}\right) \cdot f \\
& +t^{-1} f_{1} \chi^{1 / 2} \cdot\left(t^{1-\delta^{\prime}} \Gamma_{t}-\delta^{\prime} t^{-\delta^{\prime}} g_{t}\right) \cdot \chi^{1 / 2} f_{1} \\
& +O\left(t^{-\rho}\right)
\end{aligned}
$$

Now we can decompose each term of the sum (C.24) in the sense of (C.7):

Term $(k=1)$ : We introduce two quadratic forms

$$
\begin{aligned}
& B_{1}:=t^{-1} f_{1} \chi_{1} \cdot\left(\theta_{1}-t^{1-\delta^{\prime}} \Gamma_{t}\right) \cdot f_{1} \\
& B_{2}:=t^{-1} \chi_{1}^{1 / 2} f_{1} \cdot\left(\delta^{\prime} t^{-\delta^{\prime}} g_{t}-\theta_{1}\right) \cdot f_{1} \chi_{1}^{1 / 2} .
\end{aligned}
$$


Clearly

$$
\operatorname{Term}(k=1)=-B_{1}-B_{2} .
$$

We observe that $\chi_{1} \cdot\left(\theta_{1}-t^{1-\delta^{\prime}} \Gamma_{t}\right) \geq 0$ due to the support of $\chi_{1}$. Since according to Fig. 8

$$
\theta \leq t^{-\delta^{\prime}} g_{t} \leq \theta+\epsilon
$$

on $\operatorname{supp}\left(f_{1}\right)$, we also have $f_{1} \cdot\left(\delta^{\prime} t^{-\delta^{\prime}} g_{t}-\theta_{1}\right) \cdot f_{1} \geq 0$ by choosing $0<\theta_{1}<\delta^{\prime} \theta$. Hence $B_{1}$ and $B_{2}$ are positive.

Next, since $\chi_{1}^{\prime} \leq 0$ and $\left|\chi_{1}^{\prime}\right| t^{1-\delta^{\prime}} \Gamma_{t} \geq 0$ we obtain from (C.37)

$$
\begin{aligned}
D_{t}\left(f\left(\chi_{1}\right)_{t} f\right) \leq & t^{-1} f_{1} \chi_{1}^{1 / 2} \cdot\left(t^{1-\delta^{\prime}} \Gamma_{t}-\delta^{\prime} t^{-\delta^{\prime}} g_{t}\right) \cdot \chi_{1}^{1 / 2} f_{1}+O\left(t^{-\rho}\right) \\
= & -t^{-1} f_{1} \chi_{1} \cdot\left(\theta_{1}-t^{1-\delta^{\prime}} \Gamma_{t}\right) \cdot f_{1} \\
& \quad-t^{-1} \chi_{1}^{1 / 2} f_{1} \cdot\left(\delta^{\prime} t^{-\delta^{\prime}} g_{t}-\theta_{1}\right) \cdot f_{1} \chi_{1}^{1 / 2}+O\left(t^{-\rho}\right) .
\end{aligned}
$$

Now (C.39) shows that the two positive quadratic forms $B_{1}$ and $B_{2}$ are integrable in the sense of (C.8).

Term $(k=2)$ : Choosing $\theta_{2}>\delta^{\prime}(\theta+\epsilon)$ we find in the same way

$$
\begin{aligned}
\operatorname{Term}(k=2) & =B_{3}+B_{4}+O\left(t^{-\rho}\right) \\
B_{3} & :=t^{-1} f_{1} \chi_{2}^{1 / 2} \cdot\left(t^{1-\delta^{\prime}} \Gamma_{t}-\theta_{2}\right) \cdot \chi_{2}^{1 / 2} f_{1} \\
B_{4} & :=t^{-1} \chi_{2}^{1 / 2} f_{1} \cdot\left(\theta_{2}-\delta^{\prime} t^{-\delta^{\prime}} g_{t}\right) \cdot f_{1} \chi_{2}^{1 / 2}
\end{aligned}
$$

In addition we also conclude from (C.37) that

$$
D_{t}\left(f\left(\chi_{2}\right)_{t} f\right) \geq\left(1-\delta^{\prime}\right) t^{-1} f \chi_{2}^{\prime} \cdot\left(t^{1-\delta^{\prime}} \Gamma_{t}\right) \cdot f+O\left(t^{-\rho}\right)
$$

which gives

$$
\int_{1}^{\infty} d t t^{-1}\left\langle f \chi_{2}^{\prime} t^{1-\delta^{\prime}} \Gamma_{t} f\right\rangle_{t} \leq \text { const. }\|\psi\|_{H}^{2}
$$

Term $(k=3)$ : Here we exploit (C.41) and the fact that

$$
\tilde{\chi}_{2}(x):=\int_{-\infty}^{x} d s \chi_{3}(s)
$$

has the same general form as $\chi_{2}$, but with support $\left[\theta_{3},+\infty\right)$. Hence, replacing $f$ by a function $\tilde{f}$ shown in Fig. 8 such that $\theta_{3}>\delta^{\prime}(\tilde{\theta}+\epsilon)$, (C.41) takes the form

$$
\int_{1}^{\infty} d t t^{-1}\left\langle\tilde{f} \chi_{3} t^{1-\delta^{\prime}} \Gamma_{t} \tilde{f}\right\rangle_{t} \leq \text { const. }\|\psi\|_{H}^{2}
$$

Since $\tilde{\theta}<\theta$ we also have $f_{1} \leq$ const. $\tilde{f}$, so that

$$
f_{1} \chi_{3} \cdot\left(t^{1-\delta^{\prime}} \Gamma_{t}\right) \cdot f_{1} \leq \text { const. } \tilde{f} \chi_{3} \cdot\left(t^{1-\delta^{\prime}} \Gamma_{t}\right) \cdot \tilde{f}+O\left(t^{-\rho}\right) \text {. }
$$


Therefore (C.42) implies

$$
\int_{1}^{\infty} d t t^{-1}\left\langle f_{1} \chi_{3} \cdot\left(t^{1-\delta^{\prime}} \Gamma_{t}\right) \cdot f_{1}\right\rangle_{t} \leq \text { const. }\|\psi\|_{H}^{2},
$$

and since $\chi_{3} \cdot\left(t^{1-\delta^{\prime}} \Gamma_{t}\right) \geq \theta_{3} \chi_{3}$

$$
\begin{array}{r}
\int_{1}^{\infty} d t t^{-1}\left\langle f_{1} \chi_{3} f_{1}\right\rangle_{t} \leq \text { const. }\|\psi\|_{H}^{2} ; \\
\int_{1}^{\infty} d t t^{-1}\left\langle\chi_{3}^{1 / 2} f_{1} \cdot\left(t^{-\delta^{\prime}} g_{t}\right) \cdot f_{1} \chi_{3}^{1 / 2}\right\rangle_{t} \leq \text { const. }\|\psi\|_{H}^{2} .
\end{array}
$$

From this we obtain directly

$$
\begin{aligned}
\operatorname{Term}(k=3) & =B_{5}-B_{6}+O\left(t^{-\rho}\right) \\
B_{5} & :=t^{-1} f_{1} \chi_{3} \cdot\left(t^{1-\delta^{\prime}} \Gamma_{t}\right) \cdot f_{1} ; \\
B_{6} & :=t^{-1} \chi_{3}^{1 / 2} f_{1} \cdot\left(\delta^{\prime} t^{-\delta^{\prime}} g_{t}\right) \cdot f_{1} \chi_{3}^{1 / 2}
\end{aligned}
$$

with the forms $B_{5}$ and $B_{6}$ positive and integrable in the sense of (C.8). This concludes the proof of Theorem C.2.

\section{References}

[1] S. Agmon, Lectures on Exponential Decay of Solutions of Second Order Elliptic Equations, Princeton Univ. Press, 1982.

[2] W. O. Amrein, A. Boutet de Monvel and V. Georgescu, " $C_{0}$-Groups, Commutator Methods and Spectral Theory for $N$-Body Hamiltonians", Progress in Mathematical Physics 135, Birkhäuser, 1996.

[3] E. Balslev and J.-M. Combes, "Spectral properties of many-body Schrödinger operators with dilation analytic interactions", Commun. Math. Phys. 22 (1971) 280-294.

[4] A. Boutet de Monvel, V. Georgescu and A. Soffer, " $N$-body Hamiltonians with hard core interactions", Rev. Math. Phys. 6 (1994) 515-596.

[5] H. Cycon, R. Froese, W. Kirsch and B. Simon, "Schrödinger Operators", Texts and Monographs in Physics, Springer-Verlag, Berlin, Heidelberg, New York, 1987.

[6] E. B. Davies, "Spectral Theory and Differential Operators", Cambridge Univ. Press, 1995.

[7] P. Deift and B. Simon, "A time-dependent approach to the completeness of $N$-particle quantum systems", Comm. Pure Appl. Math. 30 (1977) 573-578.

[8] J. Dereziński, "Asymptotic completeness for long-range $N$-body systems. Main ideas of a proof", in Schrödinger Operators, the Quantum Mechanical Many-Body Problem, ed. E. Balslev, Lecture Notes in Physics 403 (1992) 56-72.

[9] J. Dereziński, "Asymptotic completeness for $N$-particle long-range quantum systems", Ann. Math. 138 (1993) 427-476.

[10] J. Dereziński and C. Gérard, "A remark on the asymptotic clustering of $N$-body systems", in Schrödinger Operators, the Quantum Mechanical Many-Body Problem, ed. E. Balslev, Lecture Notes in Physics 403 (1992) 73-84.

[11] J. Dereziński and C. Gérard, Scattering Theory of Classical and Quantum N-Particle Systems, Texts and Monographs in Physics. Springer-Verlag, 1997.

[12] V. Enss, "A note on Hunziker's theorem", Commun. Math. Phys. 52 (1977) 233-238. 
[13] V. Enss, "Asymptotic completeness for quantum mechanical potential scattering", Commun. Math. Phys. 61 (1978) 285-291.

[14] V. Enss, "Completeness of three-body scattering", in Dynamics and Processes, eds. P. Blanchard and L. Streit, Lecture Notes in Math. 103, 62-88, Springer Verlag, 1983.

[15] V. Enss, "Quantum scattering theory for two- and three-body systems with potentials of short- and long-range", in Schrödinger Operators, ed. S. Graffi, Lecture Notes in Mathematics, 1159, 39-178. Springer-Verlag, Berlin and New York, 1985.

[16] L. D. Faddeev, "Mathematical problems of the quantum theory of scattering for a three-particle system", Publications of the Steklov Mathematical Institute 69, Leningrad (1983) (in Russian), and Israel program for scientific translations, Jerusalem (1965).

[17] G. M. Graf, "Asymptotic completeness for $N$-body short-range quantum systems: a new proof", Commun. Math. Phys. 132 (1990) 73-101.

[18] G. M. Graf, private communication.

[19] M. Griesemer, "N-body systems with singular potentials", Ann. Inst. H. Poincaré 69 (1998) 135-187.

[20] R. Haag, "Quantum field theories with composite particles and asymptotic conditions", Phys. Rev. 112 (1958) 669-673.

[21] R. Haag, "The framework of quantum field theory", Nuovo Cim. Suppl. 14 (1959) 131-152.

[22] M. N. Hack, "Wave operators in multichannel scattering", Nuovo Cim. 13 (1959) 231-236.

[23] B. Helffer and J. Sjöstrand, "Equation de Schrödinger avec champ magnétique et équation de Harper", in Schrödinger operators, eds. H. Holden and A. Jensen, Lecture notes in Physics 345, Springer Verlag, 1989.

[24] K. Hepp, "On the quantum mechanical N-body problem", Helv. Phys. Acta 42 (1969) 425-458.

[25] W. Hunziker, "Mathematical theory of multi-particle quantum systems, Lect. Notes in Theor. Physics X, eds. A. Barut and W. Britten, Gordon and Brach, N.Y., 1968.

[26] W. Hunziker and I. M. Sigal, "The general theory of $N$-body quantum systems", in Mathematical Quantum Theory: II. Schrödinger Operators, eds. J. Feldman et al., CRM Proc. and Lecture Notes 8, Amer. Math. Soc., 1995.

[27] A. Iftimovici, "On asymptotic completeness for Agmon type Hamiltonians", $C . R$. Acad. Sci. Paris 314, Série I (1992) 337-342.

[28] V. Ivrii and I. M. Sigal, "Asymptotics of the ground state energies of large Coulomb systems", Ann. Math. 138 (2) (1993) 143-335.

[29] J. M. Jauch, "Theory of the scattering operator II, multichannel scattering", Helv. Phys. Acta 31 (1958) 661-684.

[30] R. J. Iorio, Jr. and M. O'Carroll, "Asymptotic completeness of multiparticle Schrödinger Hamiltonians with weak potentials", Commun. Math. Phys. 27 (1972) 137-145.

[31] T. Kato, "Fundamental properties of Hamiltonian operators of Schrödinger type", Trans. Amer. Math. Soc. 70 (1951) 195-211.

[32] T. Kato, "Smooth operators and commutators", Studia Math. XXXI (1968) 535-546.

[33] R. Lavine, "Commutators and scattering theory I: Repulsive interactions", Commun. Math. Phys. 20 (1971) 301-323.

[34] R. Lavine, "Completeness of the wave operators in the repulsive $N$-body problem", J. Math. Phys. 14 (1973) 376-379.

[35] E. Mourre, "Absence of singular continuous spectrum for certain self-adjoint operators", Commun. Math. Phys. 78 (1981) 391-408.

[36] E. Mourre, "Opérateurs conjugués et propriétés de propagation", Commun. Math. Phys. 91 (1983) 279-300.

[37] P. Perry, I. M. Sigal and B. Simon, "Spectral analysis of N-body Schrödinger operators", Ann. Math. 114 (1981) 519-567. 
[38] C. R. Putnam, "Commutation Properties of Hilbert Space Operators and Related Topics", Springer Verlag, 1967.

[39] M. Reed and B. Simon, Methods of Modern Mathematical Physics, Vol. I-IV, Academic Press.

[40] D. Ruelle, "On the asymptotic condition in quantum field theory", Helv. Phys. Acta 35 (1962) 147-163.

[41] D. Ruelle, "A remark on bound states in potential scattering theory", Nuovo Cimento A61 (1969) 655-662.

[42] I. M. Sigal, Mathematical Foundations of Quantum Scattering Theory for Multiparticle Systems, Memoirs of the Amer. Math. Soc. N209, 1978.

[43] I. M. Sigal, "Geometric methods in the quantum many body problem: Non-existence of very negative ions", Commun. Math. Phys. 85 (1982) 309-324.

[44] I. M. Sigal and A. Soffer, "The $N$-particle scattering problem: asymptotic completeness for short-range systems", Ann. Math. 126 (1987) 35-108.

[45] I. M. Sigal and A. Soffer, "Local decay and minimal velocity bounds", preprint, Princeton, 1988

[46] I. M. Sigal and A. Soffer, "Long-range many-body scattering. Asymptotic clustering for Coulomb-type potentials", Invent. Math. 99 (1990) 115-143.

[47] I. M. Sigal and A. Soffer, "Asymptotic completeness for $N \leq 4$ particle systems with Coulomb-type interactions", Duke Math. J. 71 (1993) 243-298.

[48] I. M. Sigal and A. Soffer, "Asymptotic completeness of $N$-Particle long range systems", J. AMS 7 (1994) 307-333.

[49] B. Simon, "Resonances in $N$-body quantum systems with dilation analytic potentials and the foundations of time-dependent perturbation theory", Ann. Math. 97 (1973) 247-274.

[50] B. Simon, "Geometric methods in multiparticle quantum systems", Commun. Math. Phys. 55 (1977) 259-274.

[51] E. Skibsted, "Propagation estimates for $N$-body Schrödinger operators", Commun. Math. Phys. 142 (1991) 67-98.

[52] H. Tamura, "Asymptotic completeness for $N$-body Schrödinger operators with shortrange interactions", Commun. P.D.E. 16 (1991) 1129-1154.

[53] D. R. Yafaev, "Radiation conditions and scattering theory for $N$-particle Hamiltonians", Commun. Math. Phys. 154 (1993) 523-554.

[54] L. Zielinski, "A Proof of asymptotic completeness for $N$-Body Schrödinger operators", Commun. P.D.E. 19 (1994) 455-522. 